\title{
On EXAFS Debye-Waller factor and recent advances
}

\section{P. Fornasini and R. Grisenti}

J. Synchrotron Rad. (2015). 22, 1242-1257

\section{IUCr Journals CRYSTALLOGRAPHY JOURNALS ONLINE}

Copyright (C) International Union of Crystallography

Author(s) of this paper may load this reprint on their own web site or institutional repository provided that this cover page is retained. Republication of this article or its storage in electronic databases other than as specified above is not permitted without prior permission in writing from the IUCr.

For further information see http://journals.iucr.org/services/authorrights.html 


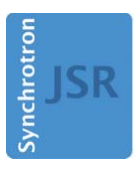

ISSN 1600-5775

OURAL OF

SYNCHROTRON

RADIATION

Received 9 February 2015

Accepted 3 June 2015

Edited by S. M. Heald, Argonne National Laboratory, USA

Keywords: EXAFS; thermal disorder; DebyeWaller factor; Einstein model; Debye model.
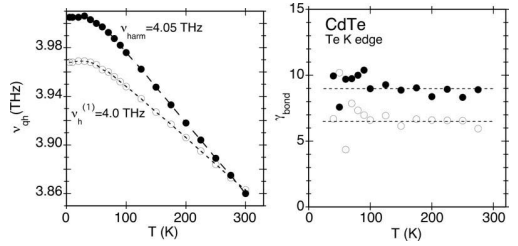

C 2015 International Union of Crystallography

\section{On EXAFS Debye-Waller factor and recent advances}

\author{
P. Fornasini* and R. Grisenti \\ Dipartimento di Fisica, Universita di Trento, I-38123 Povo, Trento, Italy. \\ *Correspondence e-mail: paolo.fornasini@unitn.it
}

The effects of structural and vibrational disorder on the EXAFS signals are parameterized in terms of the Debye Waller (DW) factor. Here the vibrational contribution is addressed, which for most systems can be singled out by studying the temperature dependence of the EXAFS DW factor, which corresponds to a good accuracy to the parallel mean square relative displacement (MSRD) around the inter-atomic equilibrium distance. By comparing the first-shell EXAFS thermal expansion with the crystallographic thermal expansion one can evaluate the perpendicular MSRD. The results of recent measurements on copper and on several tetrahedral semiconductors are here critically compared and some properties of the MSRDs are discussed, such as the dependence of correlation, force constants and vibrational anisotropy on crystal structure and bond ionicity as well as the relative merits of the correlated Debye and Einstein models. The anharmonic contribution to the parallel MSRD of CdTe has been evaluated and a quasi-harmonic analysis has been attempted, leading to an estimation of the bond Grüneisen parameter.

\section{Introduction}

EXAFS spectra are damped by the effect of disorder, of both vibrational and structural origin (Lee et al., 1981; Rehr \& Albers, 2000; Fornasini, 2015a). For each scattering path, an EXAFS experiment samples a unidimensional distribution of inter-atomic distances that, for weak disorder, can be parameterized in terms of its leading cumulants (Bunker, 1983). The second cumulant, or Debye-Waller (DW) exponent, is the variance of the distance distribution; for most systems, in the absence of phase transitions, its temperature dependence is simply connected to the vibrational properties. To a good approximation, the second cumulant corresponds to the parallel mean square relative displacement (MSRD).

An a priori knowledge of the vibrational contribution to the DW exponents can facilitate the analysis of experimental data; by converse, the results of data analyses can provide original information on local vibrational dynamics and contribute to the validation of theoretical models.

An introductory treatment of the effects of vibrational disorder on EXAFS, including the cumulant approach and a discussion of strengths and limitations on the correlated Debye and Einstein models, has been given by Dalba \& Fornasini (1997). Non-negligible advances, both theoretical and experimental, have been performed since then.

Different theoretical approaches have been attempted to evaluate the vibrational contribution to the EXAFS cumulants, and specifically to the DW factor. The method for calculating the cumulants from the force constants of the crystal potential, based on first-principles many-body perturbation theory proposed by Fujikawa \& Miyanaga (1993), has 
been thoroughly applied mainly to unidimensional systems (Miyanaga \& Fujikawa, 1994), but some attempts have been made also for face-centred cubic (f.c.c.) crystals (Katsumata $e t$ al., 2001). The connection of the EXAFS cumulants to the force constants of the effective pair potential, obtained within the framework of a quantum perturbative approach (Frenkel \& Rehr, 1993; Yokoyama, 1999), makes now possible an accurate interpretation of the low-temperature behaviour of bond expansion and the third cumulant. By a simple phenomenological approach, Van Hung \& Rehr (1997) derived an anharmonic correlated model for the effective potential taking into account the interaction of absorber and backscatterer atoms with their nearest neighbours via a Morse potential; the method was applied to calculations for $\mathrm{Cu}$ and Ni (Van Hung \& Fornasini, 2007) and for Zn and Cd (Van Hung et al., 2014a) and extended to Si and Ge using StillingerWeber potentials ((Van Hung et al., 2014b). Following the pioneering work of Benfatto and co-workers (Benfatto et al., 1989), the cumulants have been evaluated by sampling a configurational space obtained by molecular dynamics, both classical (Edwards et al., 1997; Sanson, 2010) and ab initio (Vila et al., 2012),), as well as by path-integral techniques, based on the use of effective potentials (Yokoyama, 1998; Miyanaga \& Fujikawa, 1998) or on Monte Carlo sampling (a Beccara et al., 2003; a Beccara \& Fornasini, 2008). Poiarkova \& Rehr (1999) used the equation of motion method, which involves the Fourier transform of the time dependence of the molecular dynamics, to calculate the DW factor of multiple-scattering paths in $\mathrm{Cu}$, Ge and $\mathrm{Zn}$ tetraimidazole. $A b$ initio calculations based on the density functional theory (DFT) formalism were performed for several molecules by Dimakis \& Bunker (1998); a critical evaluation of the different approximations to DFT was made by Vila et al. (2007), who further exploited the Lanczos algorithm to diagonalize the dynamical matrix.

From the experimental point of view, accurate evaluations of the bond thermal expansion, of the third cumulant and sometimes also of the fourth cumulant, have been reported (Yokoyama et al., 1997; Dalba et al., 1999; Fornasini et al., 2004; Schnohr et al., 2009; Abd el All et al., 2013). The comparison of bond and crystallographic expansions allows the evaluation of the perpendicular MSRD (Dalba et al., 1995a; Kamishima et al., 1997). From parallel and perpendicular MSRD one can reconstruct the ellipsoid of relative vibrations of nearestneighbour atoms (Ahmed et al., 2013; Fornasini, 2015b). The high accuracy of measurements has allowed the detection of the tiny isotopic effects on the low-temperature values of the parallel MSRD in germanium (Purans et al., 2008).

The EXAFS DW factor is nowadays a significant parameter of EXAFS analyses, which can be exploited to gain original information in a number of different cases, such as local mechanisms of negative thermal expansion (Bridges et al., 2014),), pressure-induced phase transitions (Principi et al., 2004; Popescu et al., 2011), structure and dynamics of nanoparticles (Ikemoto \& Miyanaga, 2007; Comaschi et al., 2008; Araujo et al., 2008) and thermally induced atomic strains (Ruffoni et al., 2007).
This paper is intended to be an updated version of the previous paper (Dalba \& Fornasini, 1997). The main advances in the theoretical description of the vibrational contribution to the DW factor are summarized in $\S 2$. Some experimental considerations are made in $\$ 3$, concerning a comparison between the ratio method and non-linear fitting procedures, the evaluation of uncertainties and the separation of the static and vibrational contributions to the DW factor. In $\$ 4-\$ 6$, recent experimental results on $\mathrm{Cu}$ and on some crystals with the diamond-zincblende structure are critically discussed: $\$ 4$ is dedicated to the harmonic analysis of the first shell, with emphasis on correlation and anisotropy as well as on strengths and limitations of the correlated Debye and Einstein models; in $\$ 5$ the anharmonic contributions to the first-shell parallel MSRD of CdTe are evaluated and an estimation of the bond Grüneisen parameter is attempted; in $\$ 6$ the correlation, the force constants and the Debye temperatures of the outer coordination shells are analysed. $\S 7$ is dedicated to conclusions.

The phenomenological approach of the previous paper is maintained here, focusing the attention only on the vibrational contribution and on the information directly obtainable from experiment. With the exception of a few seminal papers, only the literature posterior to 1997 is cited.

\section{Theoretical background}

\subsection{Unidimensional model and cumulants}

An EXAFS experiment samples a unidimensional distribution of distances for each scattering path. Owing to vibrational disorder (zero-point energy plus thermal motion) and possibly to structural disorder, the EXAFS signal is a configurational average (Tranquada \& Ingalls, 1983; Benfatto et al., 1989):

$$
\chi(k) \propto\left\langle\frac{\exp [-2 r / \lambda(k)]}{r^{2}} \exp (2 i k r)\right\rangle,
$$

where $r$ is the inter-atomic instantaneous distance and $\lambda(k)$ is the electron mean free path. Equivalently, the EXAFS signal for one scattering path is expressed, within the plane-wave approximation, as the integral (Crozier et al., 1988; Vaccari \& Fornasini, 2005)

$$
\chi(k)=\frac{S_{0}^{2}}{k} N \operatorname{Im}\left[f(k, \pi) \exp (2 i \delta) \int_{0}^{\infty} P(r, \lambda) \exp (2 i k r) \mathrm{d} r\right],
$$

where $P(r, \lambda)=\rho(r) \exp (-2 r / \lambda) / r^{2}$ is an effective distribution and $\rho(r)$ is the real distribution of distances.

Curved wave effects can be non-negligible for broad distributions $\rho(r)$; in such cases, the scattering amplitude is weakly dependent on the distance, and $f(k, \pi, r)$ should be taken under the integral of equation (2). The contributions of multiple scattering (MS) paths can be taken into account by equation (2), provided an effective amplitude of backscattering $f_{\text {eff }}(k, \pi, r)$ is considered (Rehr \& Albers, 2000). 
For moderate degrees of disorder, the effective distribution can be expanded as (Bunker, 1983; Crozier et al., 1988)

$$
\ln \int_{0}^{\infty} P(r, \lambda) \exp (2 i k r) \mathrm{d} r=\sum_{n=0}^{\infty} \frac{(2 i k)^{n}}{n !} C_{n}
$$

and the EXAFS signal can be parametrized as

$$
\begin{aligned}
k \chi(k)= & S_{0}^{2}|f(k, \pi)| N \\
& \times \exp \left[C_{0}-2 C_{2} k^{2}+\frac{2}{3} C_{4} k^{4}-\frac{4}{45} C_{6} k^{6}+\ldots\right] \\
& \times \sin \left[2 C_{1} k-\frac{4}{3} C_{3} k^{3}+\frac{4}{15} C_{5} k^{5}+\ldots+\varphi(k)\right] .
\end{aligned}
$$

$C_{n}$ are the cumulants of the effective distribution. The first and second cumulants, $C_{1}=\langle r\rangle$ and $C_{2}=\sigma^{2}=\left\langle(r-\langle r\rangle)^{2}\right\rangle$, are the average value and the variance of the effective distribution, respectively. Higher-order cumulants quantify the deviation of the distribution from the Gaussian shape; the third cumulant $C_{3}=\left\langle(r-\langle r\rangle)^{3}\right\rangle$ is a measure of the distribution asymmetry.

\subsection{Real and effective distributions}

Different procedures for connecting the cumulants of the real and of the effective distributions $\left(C_{n}^{*}\right.$ and $C_{n}$, respectively) have been proposed, limited to the first cumulant (Bunker, 1983; Freund et al., 1989) or extended to higherorder cumulants (Fornasini et al., 2001). A relatively good approximation is obtained by the recursion formula (Vaccari, 2006; Vaccari et al., 2007)

$$
C_{n}^{*} \simeq C_{n}+2 C_{n+1}\left(1 / C_{1}+1 / \lambda\right) \text { for } n=1,2,3, \ldots
$$

The difference between the first cumulants $(n=1)$ of the real and effective distributions is significant and equation (5) is included in most data analysis packages. For higher-order cumulants $(n \geq 2)$, the difference is smaller and is frequently neglected.

Let us give quantitative evaluations for two crystals considered in the next sections, $\mathrm{CdTe}$ and $\mathrm{Cu}$. The difference of first cumulants is $7.2 \times 10^{-3} \AA$ for $\mathrm{CdTe}$ at $300 \mathrm{~K}$ and $1.2 \times 10^{-3} \AA$ for $\mathrm{Cu}$ at $500 \mathrm{~K}$. The relative difference of second cumulants, evaluated through equation (5) and checked by reconstructing the distributions and evaluating their cumulants, is $1.6 \%$ for CdTe at $300 \mathrm{~K}$ and $3.5 \%$ for $\mathrm{Cu}$ at $500 \mathrm{~K}$.

\subsection{Pair potential energy}

In the unidimensional model, the vibrational properties of the absorber-backscatterer atomic pair are connected to a unidimensional pair potential energy

$$
V(u)=\frac{1}{2} k_{0} u^{2}+k_{3} u^{3}+k_{4} u^{4}+\ldots,
$$

where $u=r-r_{0}$ is the deviation of the inter-atomic distance from the position of the potential minimum.

For weak thermal disorder, the temperature dependence of the lowest-order cumulants can be expressed in terms of the force constants of the pair potential (6). In Dalba \& Fornasini
(1997), the classical approximation of Tranquada \& Ingalls (1983) and Stern et al. (1991) has been reported.

A quantum relation, based on a perturbative approach (Feynman, 1972), has been proposed by Frenkel \& Rehr (1993) and extended to second order by Yokoyama (1999). Considering the harmonic approximation as the unperturbed Hamiltonian, and defining $\omega=\left(k_{0} / \mu\right)^{1 / 2}, \sigma_{0}^{2}=\hbar / 2 \mu \omega$ and $z=\exp (-\beta \hbar \omega)$, where $\mu$ is the reduced mass, one finds (Yokoyama, 1999)

$$
\begin{aligned}
\delta C_{1}^{*} & (T)=-\frac{3 k_{3} \sigma_{0}^{2}}{k_{0}} \frac{1+z}{1-z}+\cdots, \\
C_{2}^{*}(T)=\sigma_{0}^{2} & \frac{1+z}{1-z} \\
& -\frac{12 k_{4} \sigma_{0}^{6}}{\hbar \omega} \frac{(1+z)^{2}}{(1-z)^{2}}-\frac{24 k_{4} \sigma_{0}^{6}}{k_{\mathrm{B}} T} \frac{z(1+z)}{(1-z)^{3}} \\
& +\frac{4 k_{3}^{2} \sigma_{0}^{8}}{(\hbar \omega)^{2}} \frac{13 z^{2}+58 z+13}{(1-z)^{2}} \\
& +\frac{120 k_{3}^{2} \sigma_{0}^{8}}{\hbar \omega k_{\mathrm{B}} T} \frac{z(1+z)}{(1-z)^{3}}+\cdots, \\
C_{3}^{*}(T) & =-\frac{2 k_{3} \sigma_{0}^{4}}{k_{0}} \frac{z^{2}+10 z+1}{(1-z)^{2}}-\cdots, \\
& +\frac{720 k_{3}^{2} \sigma_{0}^{10}}{\hbar \omega k_{\mathrm{B}} T} \frac{z^{2}}{(1-z)^{4}}+\cdots \\
C_{4}^{*}(T)= & -\frac{12 k_{4} \sigma_{0}^{8}}{\hbar \omega} \frac{z^{3}+9 z^{2}+9 z+1}{(1-z)^{3}} \\
& -\frac{144 k_{4} \sigma_{0}^{8}}{k_{\mathrm{B}} T} \frac{z^{2}}{(1-z)^{4}} \\
& \frac{12 k_{0}^{2} \sigma_{0}^{10}}{(\hbar \omega)^{2}} \frac{5 z^{3}+109 z^{2}+109 z+5}{(1-z)^{3}} \\
& \\
& \\
&
\end{aligned}
$$

Note that in (11) and (13) two misprints of Yokoyama (1999) have been corrected (Yokoyama, 2004).

The quantum perturbative calculations have been extended to the third order for the first, second and fourth cumulants and to the fourth order for the third cumulant by Haug et al. (2008) in order to account for the high-temperature anharmonicity effects.

If $k_{i}=0$ for $i \geq 3$ in (6) (harmonic approximation), the first term (8) of the second cumulant corresponds to the Einstein model, and the remaining terms, i.e. bond expansion (7), anharmonic corrections to the second cumulant (9)-(11) and higher-order cumulants (12)-(16), are zero.

Actually, the anharmonic contributions to the pair potential are not negligible (Eisenberger \& Brown, 1979), at least for the first coordination shell; bond expansion and third and fourth cumulants can be measured with good accuracy and are far from negligible (Dalba et al., 1999; Fornasini et al., 2004; Abd el All et al., 2013; Ahmed et al., 2013).

By comparing (7) with (8) and (12), one finds (Frenkel \& Rehr, 1993) that, to first order, the ratio 


$$
a=-\left(3 k_{3} / k_{0}\right) C_{2}
$$

should correspond to the thermal expansion $\delta C_{1}^{*}$.

The quantum expressions for cumulants (7)-(16) are equivalent to the classical expressions only at high temperature; while however the quantum approximation (8) to the second cumulant is standard practice, only quite recently has the low-temperature quantum behaviour of bond expansion and the third cumulant been measured with reasonable accuracy (Dalba et al., 1999; Abd el All et al., 2013).

\subsection{Unidimensional model and many-atomic systems}

The unidimensional model underpins the standard procedures of EXAFS data analysis. The connection of EXAFS cumulants with the physical properties of real systems is far from trivial.

Actually, only for two-atomic molecular gases have the connections between EXAFS cumulants and force constants (7)-(16) an immediate interpretation. In this case, (6) is the true pair potential energy and the thermal expansion is only due to its asymmetry: with reference to equation (17), $a=\delta C_{1}^{*}$. An experimental study of the bromine molecule $\mathrm{Br}_{2}$ has been performed by Yokoyama et al. (1996).

For many-atomic molecules and for condensed systems, the interpretation of EXAFS cumulants is by far less immediate. The main point is the relation between the three-dimensional structure of the $N$-atomic system and the unidimensional model used to parametrize the distribution $\rho(r)$ sampled by EXAFS.

While for two-atomic molecules the distribution $\rho(r)$ is only enlarged by vibrational disorder, in many-atomic systems the distribution and its cumulants can be affected not only by vibrational disorder but even by structural (static) disorder. Static contributions to the distribution $\rho(r)$ can be due to topological disorder in non-crystalline systems (Dalba et al., $1995 b$ ), different environment for bulk and surface atoms in crystalline nanostructures (Comaschi et al., 2008; Agostini et al., 2014), and so on. For many-atomic systems, the cumulants for $n \geq 2$ are the sum of a static and a vibrational (dynamic) contribution. If phase transitions are absent, one often assumes that the temperature dependence of cumulants is only due to dynamic disorder. This assumption, which is reasonable for the simple crystals considered in this paper, can be incorrect for more complex systems. A dramatic example is given by AgI-doped silver borate glasses (Sanson et al., 2013), where the short-range distribution of $\mathrm{I}-\mathrm{Ag}$ distances sampled by EXAFS is progressively depleted when temperature increases, so that the increase of the vibrational contribution to the second and third cumulants is nearly compensated by the reduction of the static contribution.

Even when only vibrational disorder is present, the potential energy $V(r)$ associated with the distribution $\rho(r)$ has a different meaning with respect to the case of a two-atomic molecule. $V(r)$ is now an effective potential energy, which depends on the statistically averaged behaviour of all the atoms in the crystal (Munstre de Leon et al., 1992) and has to be distinguished both from the single-pair interaction poten- tial energy $V(u)$ of (6) and from the total potential energy, defined in a $3 N$-dimensional configurational space (Van Hung \& Rehr, 1997; Van Hung \& Fornasini, 2007). For that reason, even for purely vibrational disorder the interpretation of the results of EXAFS analysis, based on the unidimensional model, is far from trivial.

For many-atomic systems, the bond expansion $\delta C_{1}^{*}$ is different from the quantity $a$ defined in (17); otherwise stated, the bond expansion cannot be accounted for solely by the anharmonicity of the effective pair potential measured by the third cumulant, and an additional temperature dependence of the minimum position of the effective pair potential energy has to be considered (Dalba et al., 1995a, 1998; Kamishima et al., 1997; Fornasini et al., 2004; Abd el All et al., 2012; Yokoyama \& Eguchi, 2011).

Such a shift of the potential energy, corresponding to a shift of the mode of the distribution, has been confirmed by theoretical simulations: path-integral Monte Carlo for $\mathrm{Cu}$ (a Beccara \& Fornasini, 2008) and molecular dynamics for Ge (Sanson, 2010). In both cases, the simulations show that for the first shell the asymmetry contribution (17) to thermal expansion slightly prevails, due to the relatively high values of the third cumulant, with respect to the contribution of the shift of the maximum of the distribution; for the outer shells the asymmetry contribution is much weaker, and the thermal expansion is mainly accounted for by the shift of the maximum of the distribution. This behaviour can be qualitatively understood by considering that the influence of the asymmetry of the single pair interaction potential is partially preserved in the first-shell effective potential (Van Hung \& Fornasini, 2007), and is rapidly washed out for the outer shells.

One can conclude that, while in diatomic molecules the bond expansion $\delta\langle r\rangle$ is completely determined by the distribution asymmetry, in many-atomic systems the contributions of asymmetry and potential shift are comparable for nearestneighbours distances and the potential shift neatly prevails for outer-shell distances (Fornasini et al., 2004; Abd el All et al., 2012; Ahmed et al., 2013).

On more general grounds, the relation between the anharmonicity of the total potential energy of the system and the anharmonicity of the effective pair potential energy has not yet been definitively clarified. An effort to take into account the anharmonicity effects on the vibrational density of states of many-atomic systems and its connection to EXAFS cumulants has been recently made by Vila et al. (2007).

A consequence of the collapse of the information from a three-dimensional structure into a unidimensional distribution is the difference between the average distance measured by EXAFS and the crystallographic distance, which is considered in the following sections.

Various authors have highlighted strengths and limitations of the cumulant method (Crozier et al., 1988; Dalba et al., 1993; Yang et al., 1997); Filipponi has stressed its inadequacy for systems affected by relatively strong structural disorder (Filipponi, 2001). It is thus sensible to carefully evaluate the results of the cumulant analyses. A limited number of polynomial coefficients $\tilde{C}_{n}$ is determined (frequently $n \leq 3$ ) in 
typical EXAFS analyses (Dalba \& Fornasini, 1997). The agreement of the temperature dependence of the polynomial coefficients $\tilde{C}_{n}$ with theoretical expectations (7)-(16) can be a self-consistent check of the convergence properties of the cumulants series, in order that polynomial coefficients could be considered as good estimates of the cumulants $C_{n}$ (Dalba $e t$ al., 1999; Fornasini et al., 2004). The soundness of the procedure is supported by the reproduction of experimental EXAFS cumulants by theoretical simulations (a Beccara et al., 2003; Vila et al., 2007; Sanson, 2010).

\subsection{The parallel and perpendicular MSRDs}

Let $\mathbf{R}_{0}$ be the distance between absorber and backscatterer atoms ( $a$ and $b$, respectively), ideally frozen at their rest positions, and let $\mathbf{u}_{\mathrm{a}}$ and $\mathbf{u}_{\mathrm{b}}$ be their instantaneous vibrational displacements with respect to the rest positions. The instantaneous inter-atomic distance $\mathbf{r}$ can be expressed as

$$
\mathbf{r}=\mathbf{R}_{0}+\Delta \mathbf{u}
$$

where $\Delta \mathbf{u}=\mathbf{u}_{\mathrm{b}}-\mathbf{u}_{\mathrm{a}}$ is the relative thermal displacement. It is convenient (Fornasini, 2001) to consider the projections of the relative displacement $\Delta \mathbf{u}$ along the bond direction $\Delta u_{\|}$and in the perpendicular plane $\Delta u_{\perp}$, defined by

$$
\Delta u_{\|}=\hat{R}_{0} \cdot \Delta \mathbf{u}, \quad \Delta u_{\perp}^{2}=\Delta u^{2}-\Delta u_{\|}^{2},
$$

where the short-hand notations $\Delta u^{2}$ and $\Delta u_{\|}^{2}$ correspond to $(\Delta \mathbf{u})^{2}$ and $\left[\hat{R}_{0} \cdot\left(\mathbf{u}_{\mathrm{b}}-\mathbf{u}_{\mathrm{a}}\right)\right]^{2}$, respectively.

An EXAFS spectrum corresponds to sampling a large number of instantaneous configurations, so that the quantities measured by EXAFS are expressed as canonical averages.

In crystals, the average inter-atomic distance, directly measured by the first EXAFS cumulant $C_{1}^{*}$, is (Lagarde, 1985; Dalba et al., 1995a; Stern, 1997; Fornasini et al., 2004)

$$
C_{1}^{*}=\langle r\rangle \simeq R_{\mathrm{c}}+\left\langle\Delta u_{\perp}^{2}\right\rangle / 2 R_{\mathrm{c}},
$$

where $R_{\mathrm{c}}$ is the crystallographic distance, measured by Bragg scattering experiments. The average distance $\langle r\rangle$ is always larger than the crystallographic distance $R_{\mathrm{c}}$, owing to the effect of perpendicular vibrations (Busing \& Levy, 1964; Willis \& Pryor, 1975). Accurate measurements of the bond expansion $\delta\langle r\rangle$ can now be routinely performed, and can give interesting information on the local behaviour of negative thermal expansion materials (Fornasini et al., 2009; Yokoyama \& Eguchi, 2011; Abd el All et al., 2012; Ahmed et al., 2013).

For a given scattering path, the second cumulant, or EXAFS DW exponent, is the variance of the distribution of path lengths; in particular, for single scattering (SS) paths,

$$
C_{2}^{*}=\sigma^{2}=\left\langle(r-\langle r\rangle)^{2}\right\rangle=\frac{1}{N} \sum_{\mathrm{b}}\left\langle\left(r_{\mathrm{b}}-\left\langle r_{\mathrm{b}}\right\rangle\right)^{2}\right\rangle,
$$

where the index $\mathrm{b}$ labels the $N$ backscattering atoms of the coordination shell. The second cumulant (21) gives thus information averaged over the $N$ atomic pairs corresponding to the different backscattering atoms of the shell. In general, the DW exponent measured by the second cumulants contains both static and dynamic contributions, $\sigma^{2}=\sigma_{\text {stat }}^{2}+\sigma_{\text {dyn }}^{2}$. We consider here only the dynamic contribution.

Within the harmonic approximation, one can show (Fornasini et al., 2001) that, for a given atomic pair,

$$
C_{2}^{*} \simeq\left\langle\Delta u_{\|}^{2}\right\rangle+\frac{1}{4 R^{2}}\left\{\left\langle\Delta u_{\perp}^{4}\right\rangle-\left[\left\langle\Delta u_{\perp}^{2}\right\rangle\right]^{2}\right\},
$$

where the leading contribution is the parallel MSRD $\left\langle\Delta u_{\|}^{2}\right\rangle$; the second contribution, generally negligible, is the variance of the distribution of the $\Delta u_{\perp}^{2}$ values, divided by $4 R^{2}$.

For a pair of absorber and backscatterer atoms, the parallel MSRD can be decomposed as (Beni \& Platzman, 1976)

$$
\left\langle\Delta u_{\|}^{2}\right\rangle=\left\langle\left(\hat{R} \cdot \mathbf{u}_{\mathrm{b}}\right)^{2}\right\rangle+\left\langle\left(\hat{R} \cdot \mathbf{u}_{\mathrm{a}}\right)^{2}\right\rangle-2\left\langle\left(\hat{R} \cdot \mathbf{u}_{\mathrm{b}}\right)\left(\hat{R} \cdot \mathbf{u}_{\mathrm{a}}\right)\right\rangle,
$$

where, for short, $\hat{R}=\hat{R}_{\mathrm{ab}}$. The first two terms are the independent mean square displacements (MSDs) of absorber and backscatterer atoms; they can be calculated from the anisotropic displacement parameters (ADPs) obtained by the refinement of X-rays or neutron diffraction patterns (Artioli, 2002; Kennedy, 1995). The third term, the displacement correlation function (DCF), depends on the correlation of the motion of absorber and backscatterer atoms.

A convenient measure of the degree of correlation is given by the dimensionless correlation function (Booth et al., 1995)

$$
\varphi_{\|}(T)=\frac{\left\langle\left(\hat{R} \cdot \mathbf{u}_{\mathrm{b}}\right)^{2}\right\rangle+\left\langle\left(\hat{R} \cdot \mathbf{u}_{\mathrm{a}}\right)^{2}\right\rangle-\left\langle\Delta u_{\|}^{2}\right\rangle}{2\left[\left\langle\left(\hat{R} \cdot \mathbf{u}_{\mathrm{b}}\right)^{2}\right\rangle\left\langle\left(\hat{R} \cdot \mathbf{u}_{\mathrm{a}}\right)^{2}\right\rangle\right]^{1 / 2}} .
$$

A value $\varphi_{\|}=0$ corresponds to a completely uncorrelated motion of the two atoms. Values $\varphi_{\|}=1$ and $\varphi_{\|}=-1$ correspond to atomic motions perfectly in phase and in opposition of phase, respectively.

The perpendicular MSRD $\left\langle\Delta u_{\perp}^{2}\right\rangle$ cannot be directly obtained from EXAFS spectra. It can, however, be calculated by inverting (20), provided $R_{\mathrm{c}}$ is known from Bragg scattering measurements (Dalba et al., 1999; Fornasini et al., 2004; Abd el All et al., 2012) and assuming that the vibrations are isotropic within the plane perpendicular to the bond. A perpendicular correlation function can be defined as

$$
\varphi_{\perp}(T)=\frac{\left\langle u_{\mathrm{b} \perp}^{2}\right\rangle+\left\langle u_{\mathrm{a} \perp}^{2}\right\rangle-\left\langle\Delta u_{\perp}^{2}\right\rangle / 2}{2\left[\left\langle u_{\mathrm{b} \perp}^{2}\right\rangle\left\langle u_{\mathrm{a} \perp}^{2}\right\rangle\right]^{1 / 2}},
$$

where $u_{\perp}^{2}$ are the ADPs perpendicular to the bond direction; for isotropic atomic vibrations, $u_{\perp}^{2}=(\hat{R} \cdot \mathbf{u})^{2}$. The division of $\left\langle\Delta u_{\perp}^{2}\right\rangle$ by 2 , here and in the following, projects the perpendicular MSRD along one direction.

In general, the degree of correlation is different for parallel and perpendicular MSRDs; as a consequence, the ellipsoid or relative absorber-backscatterer motion can be anisotropic even when the thermal ellipsoids of single atoms, measured by Bragg scattering, are isotropic (Fornasini et al., 2004; Fornasini, 2015b). The anisotropy ratio $\gamma=\left\langle\Delta u_{\perp}^{2}\right\rangle / 2\left\langle\Delta u_{\|}^{2}\right\rangle$ is temperature dependent (Vaccari et al., 2007); a temperatureindependent measure of anisotropy, based on the Einstein models, is introduced below. 


\subsection{Vibrational dynamics}

A general expression for the parallel MSRD of a pair of atoms a-b embedded in a system of $N$ atoms has been given by Crozier et al. (1988):

$$
\begin{aligned}
\left\langle\Delta u_{\|}^{2}\right\rangle= & \frac{\hbar}{2 \mu_{\mathrm{ab}}} \sum_{\lambda}\left|\left[\left(\frac{\mu_{\mathrm{ab}}}{m_{\mathrm{b}}}\right)^{1 / 2} \boldsymbol{\varepsilon}_{\mathrm{b}}(\lambda)-\left(\frac{\mu_{\mathrm{ab}}}{m_{\mathrm{a}}}\right)^{1 / 2} \boldsymbol{\varepsilon}_{\mathrm{a}}(\lambda)\right] \cdot \hat{R}\right|^{2} \\
& \times \frac{1}{\omega(\lambda)} \operatorname{coth} \frac{\hbar \omega(\lambda)}{2 k_{\mathrm{B}} T},
\end{aligned}
$$

where the sum is over all normal modes $\lambda$ of the real-space mass-adjusted dynamical matrix

$$
D_{\mathrm{ab}}=\frac{1}{\left(m_{\mathrm{a}} m_{\mathrm{b}}\right)^{1 / 2}} \frac{\partial^{2} V}{\partial \mathbf{u}_{\mathrm{a}} \partial \mathbf{u}_{\mathrm{b}}} .
$$

$V$ is here the total potential energy of the system, $\omega(\lambda)$ and $\boldsymbol{\varepsilon}(\lambda)$ are eigenfrequencies and normalized eigenvectors of the real-space dynamical matrix, $\mu_{\mathrm{ab}}$ is the reduced mass of the pair $\mathrm{a}-\mathrm{b}$, and the temperature dependence of the normal coordinate of each mode is

$$
\left\langle|Q(\lambda)|^{2}\right\rangle=\frac{\hbar}{2 \omega(\lambda)} \operatorname{coth} \frac{\hbar \omega(\lambda)}{2 k T}=\frac{\hbar}{2 \omega(\lambda)} \frac{1+z}{1-z}=\frac{\langle E(\lambda)\rangle}{\omega^{2}(\lambda)},
$$

where $z$ has the same meaning as in (8).

Following again Crozier et al. (1988), it is convenient to separate the geometrical from the thermal factors, defining the contribution of mode $\lambda$ to the parallel MSRD of the pair $a-b$ as

$$
p_{\mathrm{ab}}(\lambda)=\left|\left[\left(\frac{\mu_{\mathrm{ab}}}{m_{\mathrm{b}}}\right)^{1 / 2} \boldsymbol{\varepsilon}_{\mathrm{b}}(\lambda)-\left(\frac{\mu_{\mathrm{ab}}}{m_{\mathrm{a}}}\right)^{1 / 2} \boldsymbol{\varepsilon}_{\mathrm{a}}(\lambda)\right] \cdot \hat{R}\right|^{2}
$$

and the projected density of vibrational modes as

$$
\rho_{\mathrm{ab}}(\omega)=\sum_{\lambda} p_{\mathrm{ab}}(\lambda) \delta[\omega-\omega(\lambda)],
$$

so that the parallel MSRD of a given atomic pair can be expressed in terms of the projected density of states $\rho_{\mathrm{ab}}(\omega)$ (Crozier et al., 1988):

$$
\left\langle\Delta u_{\|}^{2}\right\rangle=\frac{\hbar}{2 \mu} \int_{0}^{\omega_{\max }} \frac{1}{\omega} \rho_{\mathrm{ab}}(\omega) \operatorname{coth}(\hbar \omega / 2 k T) \mathrm{d} \omega .
$$

The diagonalization of the dynamical matrix, to find eigenvalues and eigenvectors, can be a prohibitive task for large non-crystalline systems. Various approximate methods have been attempted (Rehr \& Alben, 1977; Poiarkova \& Rehr, 1999; Vila et al., 2007).

In crystals, the translational symmetry allows one to substitute the diagonalization of the real space dynamical matrix (27) with the diagonalization of a convenient sample of $\mathcal{N}$ Fourier-transformed $3 n \times 3 n$ dynamical matrices, where $\mathcal{N}$ is the number of primitive cells and $n$ is the number of atoms per primitive cell. Accordingly, (26) transforms into

$$
\begin{aligned}
\left\langle\Delta u_{\|}^{2}\right\rangle= & \frac{1}{\mathcal{N}} \frac{\hbar}{2 \mu_{\mathrm{ab}}} \\
& \times \sum_{\mathbf{q}, s} \mid\left[\left(\frac{\mu_{\mathrm{ab}}}{m_{\mathrm{b}}}\right)^{1 / 2} \mathbf{w}_{\mathrm{b}}(\mathbf{q}, s) \exp (i \mathbf{q} \cdot \mathbf{R})\right. \\
& \left.-\left(\frac{\mu_{\mathrm{ab}}}{m_{\mathrm{a}}}\right)^{1 / 2} \mathbf{w}_{\mathrm{a}}(\mathbf{q}, s)\right]\left.\cdot \hat{R}\right|^{2} \\
& \times \frac{1}{\omega(\mathbf{q}, s)} \operatorname{coth} \frac{\hbar \omega(\mathbf{q}, s)}{2 k_{\mathrm{B}} T},
\end{aligned}
$$

where the normal modes are now labelled by the wavevector $\mathbf{q}$ and the branch index $s$ and $\mathbf{w}(\mathbf{q}, s)$ are the corresponding eigenvectors. In practice, it is sufficient to evaluate eigenfrequencies and eigenvectors for a representative sample of normal modes within the first Brillouin zone.

In the harmonic approximation, the perpendicular MSRD of a crystal can be connected to the eigenvalues $\omega(\mathbf{q}, s)$ and eigenvectors $\mathbf{w}(\mathbf{q}, s)$ of the dynamical matrix by an expression similar to (32) (Vaccari \& Fornasini, 2006).

Equations (26) and (32) show that, in addition to the trivial temperature dependence, the contribution of a given pair of atoms $\mathrm{a}-\mathrm{b}$ to the total parallel MSRD depends: $(a)$ on the phase relations between the two eigenvectors $\boldsymbol{\varepsilon}_{\mathrm{a}}$ and $\boldsymbol{\varepsilon}_{\mathrm{b}}$ (or $\mathbf{w}_{\mathrm{a}}$ and $\mathbf{w}_{\mathrm{b}}$ ) of each normal mode, $(b)$ on the projections of the difference of the eigenvectors on the bond direction $\hat{R}_{\mathrm{ab}}$, and (c) in the case of crystals, it is convenient to distinguish, from the phase difference between eigenvectors, the inter-cell phase relation $\exp (i \mathbf{q} \cdot \mathbf{R})$ [as in (32)].

Finally, it is worth remembering that different dynamical matrices can exist, sharing the same eigenfrequencies but with different eigenvectors (Cochran, 1971). The reproduction of parallel and perpendicular MSRDs, experimentally obtained from EXAFS, represents a peculiar test for the phase relationships between eigenvectors obtained from model calculations or ab initio (Vila et al., 2007).

\subsection{Correlated Debye model}

Equations (26) and (32) are still of little practical use in EXAFS analyses. The vibrational contribution to the DW exponent $\sigma^{2}(T)$ is generally fitted to simple phenomenological models.

In the correlated Debye model (Beni \& Platzman, 1976; Bohmer \& Rabe, 1979; Sevillano et al., 1979),

$$
\sigma_{\mathrm{D}}^{2}(T)=\frac{3 \hbar}{\omega_{\mathrm{D}}^{3} m} \int_{0}^{\omega_{\mathrm{D}}} \mathrm{d} \omega \omega \operatorname{coth} \frac{\hbar \omega}{2 k T}\left[1-\frac{\omega_{\mathrm{D}} \sin \left(\omega R q_{\mathrm{D}} / \omega_{\mathrm{D}}\right)}{\omega R q_{\mathrm{D}}}\right] .
$$

$m$ is the average atomic mass of the a-b pair and the Debye frequency $\omega_{\mathrm{D}}$ is the only free parameter, corresponding to a Debye temperature $\Theta_{\mathrm{D}}=\hbar \omega_{\mathrm{D}} / k_{\mathrm{B}}$. The quantity $q_{\mathrm{D}}$ is the radius of a Debye sphere,

$$
q_{\mathrm{D}}=\left(6 \pi^{2} / V_{\mathrm{a}}\right)^{1 / 3}
$$

where $V_{\mathrm{a}}$ is the real-space volume per atom. 
Equation (33) is an extension of the Debye model for atomic vibrations in crystallography (Willis \& Pryor, 1975): the second term in square parentheses takes into account the effect of correlation due to the inter-cell phase relation $\exp (i \mathbf{q} \cdot \mathbf{R})$ in (32); no account is given of the phase relations between different eigenvectors nor of their possible different projections along the bond directions.

The correlated Debye model was originally developed for close-packed crystals with one atom per primitive cell (primitive crystals), where $V_{\mathrm{a}}$ is the volume of the primitive cell, all atoms share the same eigenvectors and close-packing ensures a high degree of isotropy of the eigenvector projections. For primitive crystals, one can reasonably expect that the EXAFS Debye temperatures are similar for different coordination shells and comparable with the Debye temperatures from other experimental techniques.

For crystals with more than one atom per primitive cell (non-primitive crystals), the usual choice (34) for the Debye wavevector corresponds to considering an extended Brillouin zone scheme, where all vibrational properties are described by three acoustic modes. The actual presence of optical modes, the intra-cell eigenvector phaseshifts and the local anisotropy connected with the open structure make, however, questionable the physical significance of the Debye model for nonprimitive crystals.

The influence of optical modes should not be overemphasized, since the amplitude of atomic vibrations is proportional to the inverse of the squared frequency. An interesting counter-example is $\beta$-AgI (Dalba et al., 1990; Dalba \& Fornasini, 1997), where a low-frequency $(0.5 \mathrm{THz})$ optical branch is present and the DW experimental behaviour was satisfactorily reproduced by a mixed model: correlated Debye (reduced Brillouin zone scheme) + Einstein. AgI is also a good example of the effect of the projection of eigenvectors along the bond directions: the contribution of the $0.5 \mathrm{THz}$ mode at the Brillouin zone centre to the first coordinations shell DW is zero, since three of the four atoms move in phase with the central atom, the fourth atom moves out of phase but the atomic displacements are normal to the inter-atomic distance.

A correlated Debye model can be fitted to the perpendicular MSRD $\left\langle\Delta u_{\perp}^{2}\right\rangle$ too (Vaccari \& Fornasini, 2006), the only difference with respect to (33) being that the prefactor is now $6 h / \omega_{\mathrm{D}}^{2}$, to account for the different dimensionality; the parallel and perpendicular Debye frequencies are generally different.

\subsection{Einstein model}

The Einstein model (Sevillano et al., 1979)

$$
\sigma_{\mathrm{E}}^{2}(T)=\frac{\hbar}{2 \mu \omega_{\mathrm{E}}} \operatorname{coth}\left(\frac{\hbar \omega_{\mathrm{E}}}{2 k T}\right)
$$

where $\mu$ is the reduced mass of the $a-b$ pair, is intrinsically correlated. The best-fitting Einstein frequencies $\omega_{\mathrm{E}}=2 \pi v_{\mathrm{E}}$ of different coordination shells are generally different even for primitive crystals.

To the Einstein frequency $\omega_{\mathrm{E}}=\omega_{\|}$an effective parallel force constant is associated, $k_{\|}=\mu\left(\omega_{\|}\right)^{2}$, which corresponds to $k_{0}$ in (6). The effective unidimensional potential energy (6) depends on the statistically averaged influence of all the atoms, so that $k_{0}$ cannot be identified with the force constant of a single-bond potential (Van Hung \& Fornasini, 2007).

The effective force constant $k_{\|}$is a measure of the interaction strength. The frequency $\omega_{\mathrm{E}}=\left(k_{\|} / \mu\right)^{1 / 2}$ represents the response to the interaction force, and depends on the inertia, measured by the reduced mass. At high temperatures, the Einstein model approaches the classical value $\sigma_{\infty}^{2}=k_{\mathrm{B}} T / k_{\|}$, independent of mass. For $T \rightarrow 0$, however, $\sigma_{\mathrm{E}}^{2} \rightarrow \hbar / 2\left(\mu k_{\|}\right)^{1 / 2}$ and depends on the reduced mass. The tiny mass effect on the low-temperature DW exponent has been recently detected by EXAFS measurements on the isotopes 70 and 76 of germanium below $150 \mathrm{~K}$ (Purans et al., 2008).

In the Einstein model, the atomic pair is treated as a single quantum oscillator. Actually, the sum (26) of the contributions of all normal modes is Einstein-like only in the hightemperature classical limit. The discrepancy between the MSRDs calculated from lattice dynamics and the best-fitting Einstein model has been recently estimated for different crystalline structures (Sanson, 2008). The discrepancy, which increases with the width of the density of vibrational states, has been found no larger than 5\% for the parallel MSRD.

An Einstein model can be fitted to the temperature dependence of the perpendicular MSRD $\left\langle\Delta u_{\perp}^{2}\right\rangle$ too (Vaccari \& Fornasini, 2006),

$$
\left\langle\Delta u_{\perp}^{2}\right\rangle=\frac{\hbar}{\mu \omega_{\perp}} \operatorname{coth}\left(\frac{\hbar \omega_{\perp}}{2 k T}\right),
$$

the only difference with respect to (35) being again a factor of two that accounts for the different dimensionality. Parallel and perpendicular Einstein frequencies are in general different. The perpendicular Einstein frequency can be connected to an effective perpendicular force constant $k_{\perp}=\mu\left(\omega_{\perp}\right)^{2}$.

The effective force constants $k_{\|}$and $k_{\perp}$ should not be confused with the force constants of lattice dynamical models. A comparison of the EXAFS effective force constants with the force constants $k_{r}$ and $k_{\theta}$ of a valence force field (VFF) model (Keating, 1966) that Martin had extended to ionic systems with the zincblende structure (Martin, 1971) has been performed for $\mathrm{Ge}, \mathrm{CdTe}$ and $\mathrm{CuCl}$ by Abd el All et al. (2012). For Ge, $k_{\|}$is $12 \%$ larger than $k_{r}$ and $k_{\perp}$ is about six times larger than $k_{\theta}$. When the ionicity increases, the decrease of $k_{\|}$ is stronger than the decrease of $k_{r}$, and $k_{\perp}$ is always about six times larger than $k_{\theta}$.

The ratio of parallel to perpendicular effective force constants, $\xi=k_{\|} / k_{\perp}$, represents a temperature-independent measure of the anisotropy of the relative vibrations, since it corresponds to the asymptotic behaviour of $\gamma=\left\langle\Delta u_{\perp}^{2}\right\rangle / 2\left\langle\Delta u_{\|}^{2}\right\rangle$ for $T \rightarrow \infty$ (Ahmed et al., 2009). For perfectly isotropic relative vibrations, $\xi=1$. 


\section{Experimental details}

After some general methodological considerations presented in this section, in the following sections $\$ 4, \S 5$ and $\S 6$ we illustrate the connection between dynamical parameters extracted from EXAFS (correlation functions, force constants, Einstein and Debye temperatures) and physical properties (such as crystal structure and bond ionicity) by comparing the results obtained for copper (Fornasini et al., 2004) and for a series of crystals with the diamond-zincblende structure with different degrees of ionicity: germanium (Dalba et al., 1999), GaAs (Ahmed et al., 2013), InP (Schnohr et al., 2009), CdTe (Abd el All et al., 2012) and $\mathrm{CuCl}$ (Vaccari et al., 2007).

\subsection{Ratio method and non-linear fit}

Only a few comments can be added to the previous discussions of the relative merits of the two methods (Fornasini et al., 2004; Vaccari et al., 2007).

The ratio method (Bunker, 1983; Dalba et al., 1993) consists of the separate analysis of phase and amplitude of the EXAFS signal at each temperature, taking a low-temperature spectrum as reference. The method is particularly suited to studying temperature-dependent variations of the first-shell parameters, since it is largely independent of theoretical inputs and highly insensitive to the correlation between even and odd cumulants; besides, it allows a direct, although sometimes quite conservative, estimation of the quality of experimental data (Abd el All et al., 2013). Care must be taken to check that the first-shell contribution can be well singled out at all temperatures by Fourier filtering and is not perturbed by leakages from outer shells (Schnohr et al., 2014).

In the second method, backscattering amplitudes, phase shifts and inelastic terms are calculated by a suitable theoretical code and a non-linear best-fit of calculated to experimental spectra is performed; in the examples presented here, the FEFF6 (Rehr et al., 1992; Ankudinov et al., 1998) and IFEFFIT (Newville, 2001) codes were used through the graphical interface Artemis (Ravel \& Newville, 2005). This procedure is the only one possible when single-shell SS contributions cannot be disentangled and/or are mixed with MS contributions, which typically happens for the second and outer shells and for mixed first shells. The procedure gives absolute values of parameters, whose reliability, however, depends on the accuracy of theoretical calculations.

The separate analysis of the outer shells contributions by the ratio method is in principle unsuitable; in some cases, however, the temperature dependence of the DW factors has been found in reasonable agreement with that obtained from the procedure based on MS calculations and non-linear fit (Dalba \& Fornasini, 1997).

\subsection{Evaluation of uncertainties}

The relevance of a sound evaluation of the accuracy of EXAFS results can hardly be overestimated. Developing effective standardization criteria for the uncertainty assessment (Ascone et al., 2012) is, however, far from trivial, in view of the large number of variable physical conditions and parameters that can affect both experiment and data analysis.

Assessing the uncertainty of EXAFS results requires a careful evaluation of random fluctuations and systematic errors in both experimental and data analysis procedures. The analysis of the different causes of uncertainty in temperaturedependent EXAFS measurements on CdTe has been made recently (Abd el All et al., 2013). Let us here only review the main points.

Temperature fluctuations of the sample and/or the optical apparatus, monochromator calibration and resolution, electron beam fluctuations, not to speak of systematic errors (related to sample temperature calibration, photon beam energy calibration, sample deterioration, and so on), cannot always be under complete control of the synchrotron radiation users. Suitable experimental strategies and data analysis procedures, joined to a critical discussion of results, can in any case lead to a sound a posteriori evaluation of uncertainties and of their most relevant causes.

EXAFS measurements at a given temperature should be repeated at least three times and each spectrum should be separately analysed. The resulting cumulants can be considered a restricted sample of a Gaussian parent population and it is reasonable to evaluate their uncertainty as the standard deviation of the distribution of mean values; this contribution to uncertainty decreases when the number of spectra increases.

As far as the data analysis is concerned, different windows and $k$ weights in Fourier transform and back-transform, as well as different fitting intervals, lead to different values of cumulants, which cannot be considered as independent samples of a parent distribution: increasing the number of fitting intervals cannot decrease the final uncertainty. The different values can be considered as sampling a uniform distribution, whose standard deviation is $\sigma_{n}=\Delta\left(\delta C_{n}\right) / \sqrt{12}$. The point here is a sound choice of the width of the uniform distribution, which to a good extent depends on skill and physical insight of the experimenter. The ratio method can be of help, when applicable, since it allows a direct visual inspection of the phase differences and of the logarithms of amplitudes ratios.

A further contribution to the evaluation of uncertainty is the comparison of measurements performed on the same system in different laboratories or on samples of different thicknesses or at two different absorption edges.

It is a common experience that, of the different parameters measured by EXAFS, the second cumulant is affected by the smallest relative uncertainties.

\subsection{Static and vibrational contributions to DW exponent}

In general, both static and vibrational disorder can contribute to the damping of the EXAFS signal, so that $\sigma^{2}=\sigma_{\text {stat }}^{2}+$ $\sigma_{\text {dyn }}^{2}$. Disentangling the two contributions can be far from trivial. For most simple systems, it seems reasonable to assume that the temperature dependence of $\sigma^{2}$ only depends on the vibrational contribution. However, in some systems the 
static contribution may exhibit temperature dependence too; an interesting example is the case of AgT-doped fast ion conduction glasses (Sanson et al., 2013), already cited in $\$ 2$.

In the following sections, we assume that the static contribution to the DW exponent of the considered crystals is temperature independent, and attribute the temperature dependence only to the vibrational contribution.

If the analysis is performed by the ratio-method, only relative values $\delta \sigma^{2}$ with respect to a reference temperature can be obtained; no evaluation of the static contribution is possible. The absolute values of $\sigma_{\text {dyn }}(T)$ are evaluated by fitting an Einstein or a Debye model to the temperature dependence $\delta \sigma^{2}(T)$ of the relative values.

If the analysis is performed by non-linear fit of theoretical simulations to experimental spectra, absolute values of $\sigma^{2}=$ $\sigma_{\text {stat }}^{2}+\sigma_{\text {dyn }}^{2}(T)$ are obtained, whose accuracy, however, depends on the accuracy of calculated backscattering amplitudes, phase-shifts and inelastic terms. In order to reduce the effects of statistical correlations, it is good practice to leave free the energy origin $e_{0}$ and the inelastic factor $S_{0}^{2}$ only in a first trial analysis and to consider their average values as fixed in the final analysis. The average values $S_{0}^{2}=0.86$ and $S_{0}^{2}=1$ were found for $\mathrm{Cu}$ and for $\mathrm{CdTe}$, respectively (Fornasini et al., 2004; Abd el All et al., 2012).

Separating the static and the vibrational contributions to $\sigma^{2}$ is in any case not immediate. In general one assumes that the Einstein or Debye models are reasonably good estimates of $\sigma_{\text {dyn }}^{2}(T)$ : both models tend to the classical harmonic behaviour at high temperature and anharmonicity contributions can in principle be taken into account by suitable corrections to the Einstein model. For the crystals considered in the following sections, the absolute values of $\sigma^{2}=\sigma_{\text {stat }}^{2}+\sigma_{\text {dyn }}^{2}(T)$ determined from the $F E F F+F E F F I T$ analysis have been found in good agreement or slightly lower that the Einstein or Debye models best fitting their temperature dependence, suggesting the negligibility of $\sigma_{\text {stat }}^{2}$.

\section{First-shell MRSD results, harmonic analysis}

More accurate values of cumulants can generally be obtained for the first shell than for the outer shells, thanks to the absence of MS contamination and to the possibility of comparing the results of both the ratio method and the nonlinear fitting procedure. Besides, the possibility of directly measuring the bond expansion $\delta C_{1}^{*}$ with good accuracy leads to the evaluation of the perpendicular MSRD.

Absolute values of the vibrational contributions to both parallel and perpendicular MSRDs are generally obtained by fitting an Einstein model to the temperature dependence of the experimental values. For the parallel MSRD, this corresponds to using only the first term (8) of the quantum perturbative expansion.

As an example of the degree of accuracy presently attainable, Fig. 1 shows the case of $\mathrm{CdTe}$, for which independent measurements were performed at both the $\mathrm{Cd}$ and Te $K$-edges (Abd el All et al., 2012, 2013).

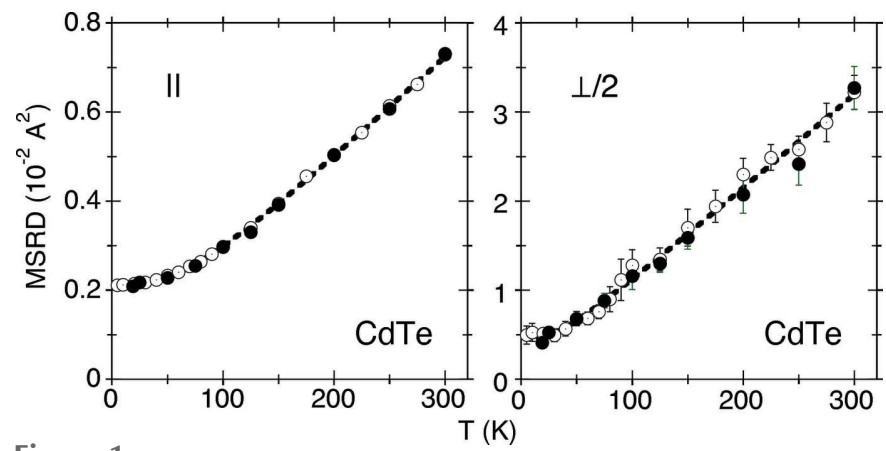

Figure 1

Parallel (left panel) and half-perpendicular (right panel) MSRDs for the first shell of CdTe (notice the different vertical scales). Solid and open symbols are the results from $\mathrm{Cd}$ and $\mathrm{Te} K$-edges, respectively; the dashed lines are best-fitting Einstein models. Data from Abd el All et al. (2012).

\subsection{Parallel and perpendicular correlation}

The values of the parallel and perpendicular correlation functions (24) and (25) evaluated at $300 \mathrm{~K}$ for the first coordination shell of $\mathrm{Cu}, \mathrm{Ge}, \mathrm{GaAs}, \mathrm{InP}, \mathrm{CdTe}$ and $\mathrm{CuCl}$ are shown in Fig. 2 as a function of the bond ionicity.

The accuracy of the correlation functions depends on the accuracy of the EXAFS MSRDs and of the atomic MSDs. The EXAFS results here presented for $\mathrm{Cu}$, Ge, GaAs and CdTe can be considered more accurate than for $\mathrm{InP}$ and $\mathrm{CuCl}$. The perpendicular MSRD is obtained, according to equation (20), by comparing the thermal expansions measured by EXAFS and Bragg scattering; when possible, EXAFS and scattering measurements should be made on the same sample (Sanson et al., 2006). Different Bragg scattering results can be found in the literature for the same system, the discrepancy being due to sample variations or perhaps to experimental errors; a nonnegligible discrepancy has been found for GaAs, leading to a non-negigible difference in perpendicular MSRD values (Ahmed et al., 2013). Experimental MSD data, obtained from the refinement of scattering patterns, are available in the literature for $\mathrm{Cu}, \mathrm{CdTe}, \mathrm{InP}$ and $\mathrm{CuCl}$ (Martin \& O'Connor, 1977; Day et al., 1995; Horning \& Staudenmann, 1986; Sara-

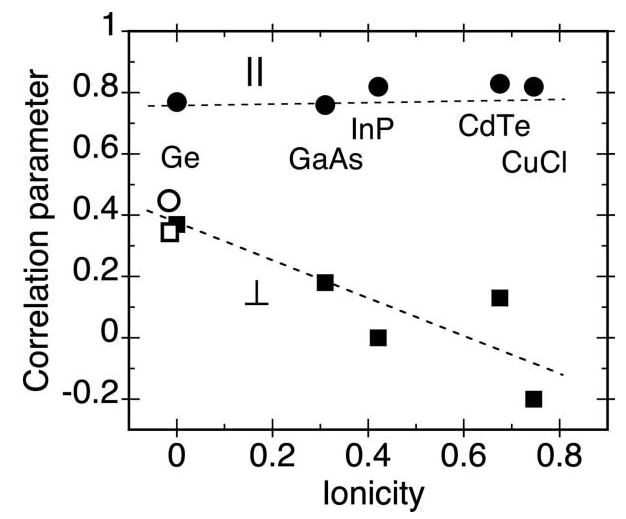

Figure 2

Values of the parallel (circles) and perpendicular (squares) correlation function evaluated at $300 \mathrm{~K}$ for the first shell of different systems: open symbols refer to $\mathrm{Cu}$, solid symbols to $\mathrm{Ge}, \mathrm{GaAs}, \mathrm{InP}, \mathrm{CdTe}$ and $\mathrm{CuCl}$. The dashed lines are guides to the eye. The references for this and for the following figures are given at the beginning of $\S 3$. 
vanan et al., 1992; Altorfer et al., 1994). For GaAs and Ge, no temperature-dependent MSD experimental values are available, and we referred to calculated values (Reid, 1983; Soma \& Matsuo, 1982), which, however, significantly depend on the lattice-dynamical model.

In spite of the difficulty of assessing the degree of accuracy for the correlation parameters of Fig. 2, some general trends can in any case be observed.

The value 0.46 of the parallel correlation for $\mathrm{Cu}$ (open circle) can be compared with the value 0.32 found for $\mathrm{Ni}$ from the analysis of total scattering patterns (Jeong et al., 1999). The parallel correlation values calculated for several f.c.c. metals ( $\mathrm{Al}, \mathrm{Ca}, \mathrm{Ni}, \mathrm{Au}$ and $\mathrm{Ce}$ ) using the BvK force model (Jeong et al., 2003), with parameters derived from phonon dispersion curves, vary from 0.37 to 0.45 .

The parallel correlation is significantly larger for the tetrahedral semiconductors (solid circles) than for copper and seems nearly independent of the bond ionicity; the values found by EXAFS vary from 0.76 to 0.82 and are consistent with the value 0.82 found for InAs from the analysis of total scattering (Jeong et al., 1999).

For both $\mathrm{Cu}_{2} \mathrm{O}$ and $\mathrm{Ag}_{2} \mathrm{O}$, where the metal atom is linearly coordinated to two oxygen atoms, the value of the correlation parameter was found to be about 0.97 (Sanson et al., 2006).

It is reasonable to conclude that the first-shell parallel correlation significantly depends on the structure: it is smaller for close-packed structures and increases with the decreasing of the coordination number. Actually, a strong correlation of the absorber atom with all its nearest-neighbours is incompatible with a large coordination number. The dependence of the parallel correlation on the interaction for a given structure, for example on the degree of ionicity for the diamond-zincblende structure, is comparably negligible.

The perpendicular correlation is only slightly smaller than the parallel correlation for copper (open square). For tetrahedral semiconductors, the perpendicular correlation (solid squares) is instead much smaller than the parallel correlation; besides, it decreases when the bond ionicity increases, indicating that it is significantly dependent on the interaction.

\subsection{Effective force constants}

The parallel and perpendicular effective force constants, calculated from the Einstein frequencies, are shown in Fig. 3.

The parallel force constant $k_{\|}$, which measures the effective bond strength, is more than twice as large in Ge (covalent bond) than in $\mathrm{Cu}$ (metallic bond); it, however, significantly decreases when the ionicity increases in tetrahedral semiconductors, and for $\mathrm{CuCl}$ it is smaller than that for $\mathrm{Cu}$. Contrary to the parallel correlation, the parallel force constant is strongly dependent on the interaction even for systems sharing the same structure.

The perpendicular force constant $k_{\perp}$ of copper is only slightly smaller than the parallel force constant. For tetrahedral semiconductors, $k_{\perp}$ is instead much smaller than $k_{\|}$, the relatively open structure favouring the perpendicular relative vibrations. Like $k_{\|}$, the perpendicular force constant also

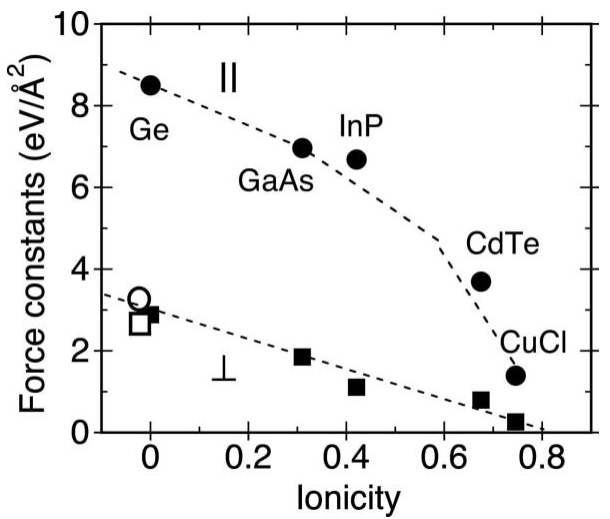

Figure 3

Parallel (circles) and perpendicular (squares) effective force constants for the first shell of different systems: open symbols refer to $\mathrm{Cu}$, solid symbols to $\mathrm{Ge}, \mathrm{GaAs}$, InP, $\mathrm{CdTe}$ and $\mathrm{CuCl}$. The dashed lines are guides to the eye.

decreases when ionicity increases, although with a slightly different trend. In any case, the values of both parallel and perpendicular force constants for $\mathrm{CuCl}$ are less than $20 \%$ of the corresponding values for Ge.

\subsection{Anisotropy}

A simple measure of the perpendicular to parallel anisotropy of relative vibrations of neighbouring atoms, $\left\langle\Delta u_{\perp}^{2}\right\rangle / 2\left\langle\Delta u_{\|}^{2}\right\rangle$, is given by the ratio of the effective force constants, $\xi=k_{\|} / k_{\perp}$ (Ahmed et al., 2009).

A direct physical insight on the extent of relative vibrations is given by the standard deviations $\sigma_{\|}=\left[\left\langle\Delta u_{\|}^{2}\right\rangle\right]^{1 / 2}$ and $\sigma_{\perp}=$ $\left[\left\langle\Delta u_{\perp}^{2}\right\rangle / 2\right]^{1 / 2}$, which directly measure the size of the relative thermal ellipsoids. The anisotropy is thus more effectively measured by $\xi^{1 / 2}=\left(k_{\|} / k_{\perp}\right)^{1 / 2}$ than by $\xi$. The values of the anisotropy parameter $\xi^{1 / 2}$ are shown in Fig. 4.

For copper, $\xi^{1 / 2} \simeq 1.1$, relative thermal vibrations are nearly isotropic. For the more open structure of tetrahedral semiconductors, the parameter $\xi^{1 / 2}$ is significantly larger; the average trend is to increase when ionicity increases, from about 1.8 for $\mathrm{Ge}$ to about 2.3 for $\mathrm{CuCl}$ : the scattering of data

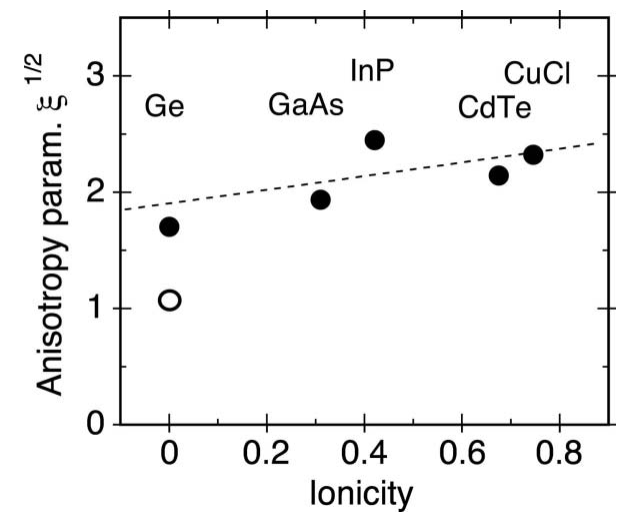

Figure 4

Anisotropy parameter $\xi^{1 / 2}=\left(k_{\|} / k_{\perp}\right)^{1 / 2}$ : the open circle is for $\mathrm{Cu}$, the solid circles are for $\mathrm{Ge}, \mathrm{GaAs}, \mathrm{InP}, \mathrm{CdTe}$ and $\mathrm{CuCl}$. The dashed line is a guide to the eye. 
with respect to a smooth behaviour can be correlated to the different mass ratios of absorber and back scatterer atoms for the different compounds (Fornasini, 2015b).

The extent of anisotropy of relative vibrations has been recently correlated to the strength of the negative lattice thermal expansion (Sanson et al., 2006; Ahmed et al., 2013).

\subsection{Einstein and Debye models}

In general, both correlated Debye and Einstein models satisfactorily fit the temperature dependence of parallel MSRD. The two models differ in the low-temperature region, and tend to the same classical behaviour $\langle E\rangle / k_{0}=k_{\mathrm{B}} T / k_{0}$ at high temperature, where, however, experimental data can be affected by anharmonicity. The similarity of the two models can be understood by considering that the DW exponent is less sensitive than specific heat to the details of the vibrational densities of states: the DW is proportional to the stored energy, the specific heat is proportional to its derivative.

The residual discrepancy between the absolute values of the correlated Debye and Einstein models best fitting the temperature dependence of the parallel MSRD of Ge was pointed out by Dalba \& Fornasini (1997). The availability of more refined data allows us now to gain a deeper insight. Let us focus on $\mathrm{Cu}$ (Fornasini et al., 2004) and CdTe (Abd el All et al., 2012). In Fig. 5, the corresponding Einstein and correlated Debye models best fitting the temperature dependence of the parallel MSRD are shown (left and right panels, respectively), for different intervals of fit.
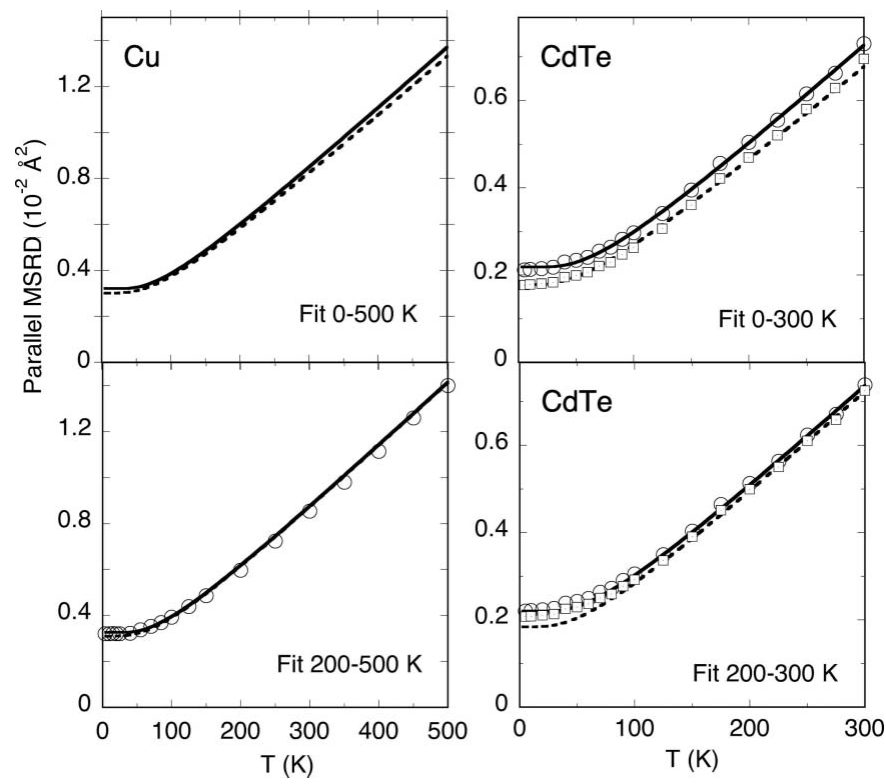

Figure 5

Einstein models (continuous lines) and correlated Debye models (dashed lines) best fitting the experimental temperature dependence of the firstshell parallel MSRD of $\mathrm{Cu}$ (left) and CdTe (right). Upper and lower panels refer to fits performed over the entire temperature range or over a limited higher temperature range, respectively. Open circles and open squares are absolute values of experimental data obtained by fitting an Einstein or a Debye model, respectively.
For copper, the experimental data extend from liquidhelium temperature up to $500 \mathrm{~K}$. When the fit is performed over the entire temperature range $0-500 \mathrm{~K}$, the Einstein frequency is $v_{\mathrm{E}}=4.96 \mathrm{THz}$ (Einstein temperature $\theta_{\mathrm{E}}=238 \mathrm{~K}$ ) and the Debye temperature is $\theta_{\mathrm{D}}=328 \mathrm{~K}$. The absolute MSRD values determined by Debye are slightly lower than those determined by Einstein, the relative discrepancy decreasing from about $7 \%$ at low temperatures to about $3 \%$ at high temperatures (Fig. 5, top left panel).

When the fit is limited to the higher-temperature region $200-500 \mathrm{~K}$, the Einstein frequency decreases by $1.8 \%$ and the Debye temperature decreases by $3.5 \%$. The two models are now in quite fair agreement at high temperature; the relative discrepancy is still about $5 \%$ at low temperatures, but decreases to less than $0.2 \%$ at high temperatures (Fig. 5, bottom left panel).

For CdTe, the experimental data extend from liquid-helium temperature up to $300 \mathrm{~K}$. When the fit is performed over the entire temperature range $0-300 \mathrm{~K}$, the Einstein frequency is $v_{\mathrm{E}}=3.87 \mathrm{THz}\left(\theta_{\mathrm{E}}=186 \mathrm{~K}\right)$ and the Debye temperature is $\theta_{\mathrm{D}}=$ $224 \mathrm{~K}$. The absolute MSRD values determined by Debye are significantly lower than those determined by Einstein, the relative discrepancy being about $23 \%$ at low temperatures and about $8 \%$ at high temperatures (Fig. 5, top right panel). The non-negligible difference at high temperature can be attributed to the influence of the low-temperature region on the fit.

When the fit is limited to the higher-temperature region 200-300 K, where both models approach the classical behaviour, the Einstein frequency decreases by $0.7 \%$ and the Debye temperature decreases by $3.5 \%$. The relative discrepancy actually decreases to about $2 \%$ at high temperatures but is still about $20 \%$ at low temperatures. The temperature dependence of the experimental data is well reproduced by both models in the high-temperature region but is better reproduced by the Einstein model in the low-temperature region.

The better agreement between the two models when the fitting interval is limited to higher temperatures (classical limit) is expected. Less obvious is the fact that when the temperature interval is reduced the absolute values of the Debye model undergo a larger modification than the absolute values of the Einstein model; this effect is much more significant for $\mathrm{CdTe}$ than for $\mathrm{Cu}$. These results suggest that the Einstein model could be preferable to the Debye model for non-primitive crystals, such as CdTe or Ge.

For non-primitive crystals, one could fit the experimental data to a mixed model, Debye (with reduced Brillouin zone) to account for acoustic modes and Einstein to account for optic modes. Actually, such a fit is highly unstable, in view of the strong similarity between the two models, which leads to a strong correlation between Einstein frequency and Debye temperature. In any case, it is interesting that the DW of CdTe is well reproduced by summing a Debye model with the diffraction Debye temperature 143 K (Zubík \& Valvoda, 1975; Stewart, 1983) and an Einstein model with a frequency centred on the peak of optic modes in the vibrational density of states (Talwar \& Holliday, 1999). 


\section{First-shell results, anharmonicity effects}

In principle, the anharmonic contributions to the second cumulant can be non-negligible. A procedure for their evaluation, based on the classical approximation, was proposed by Dalba \& Fornasini (1997) for the first three coordination shells of Ge.

In the quantum perturbative approach, the anharmonic contributions are given by equations (9)-(11). A direct fit of the sum of the terms from (8) to (11), considering $\omega, k_{3}$ and $k_{4}$ as free parameters, is highly unstable, in view of the very similar temperature dependence of the different terms. An alternative recursive procedure is here proposed, based on the use of all quantum relations (8)-(16); as a model system, we consider again the first shell of CdTe (Abd el All et al., 2012).

\subsection{Anharmonic contributions to the DW factor}

Once the Einstein frequency $v_{\mathrm{E}}$ has been determined by fitting the harmonic expression (8) to the experimental values of the second cumulant, the first step of the recursive procedure is the analysis of the third and fourth cumulants. At first, equation (12) is fitted to the experimental values of the third cumulant, the only free parameters being the third-order force constant $k_{3}$ and a vertical normalization constant. The case of the Te $K$-edge of CdTe is shown in the left panel of Fig. 6, where $k_{3} \simeq-2.07 \mathrm{eV}^{-3}$ and the non-zero low-temperature value is about $4.2 \times 10^{-6} \AA^{3}$. The fourth cumulant is then considered: the two terms (15) and (16) depend on $k_{3}$, and are thus fully determined; as one can see in the right panel of Fig. 6, for CdTe they represent a large fraction of the fourth cumulant; only the tiny remaining part depends on the free parameter $k_{4}$ of the two terms (13) and (14).

Once the force constants $k_{3}$ and $k_{4}$ have been determined, one fits the sum of all the terms (8)-(11) to the experimental temperature dependence of the second cumulant, obtaining a new Einstein frequency $v_{\mathrm{h}}^{(1)}$. The analysis of third and fourth cumulants is repeated with the new frequency $v_{\mathrm{h}}^{(1)}$, obtaining new values of force constants $k_{3}^{(1)}$ and $k_{4}^{(1)}$. The loop of fits is repeated obtaining a sequence of frequencies $v_{\mathrm{h}}^{(n)}$ which converge to a final value $v_{\text {harm }}$. For the Te edge of CdTe we
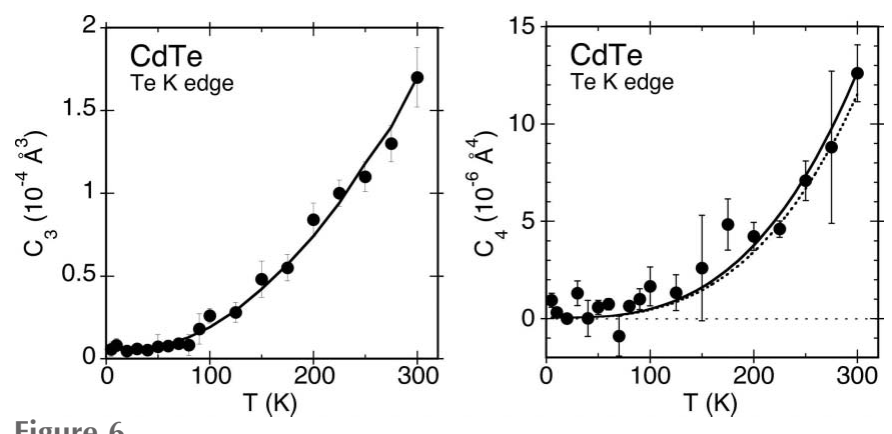

Figure 6

From EXAFS at the Te $K$-edge of CdTe (Abd el All et al., 2013). Left panel: third cumulant, the continuous line is the best fitting function (12). Right panel: fourth cumulant, the continuous line is the total fitting function, the dashed line is the contribution of the two terms (15) and (16) that depend on $k_{3}$.

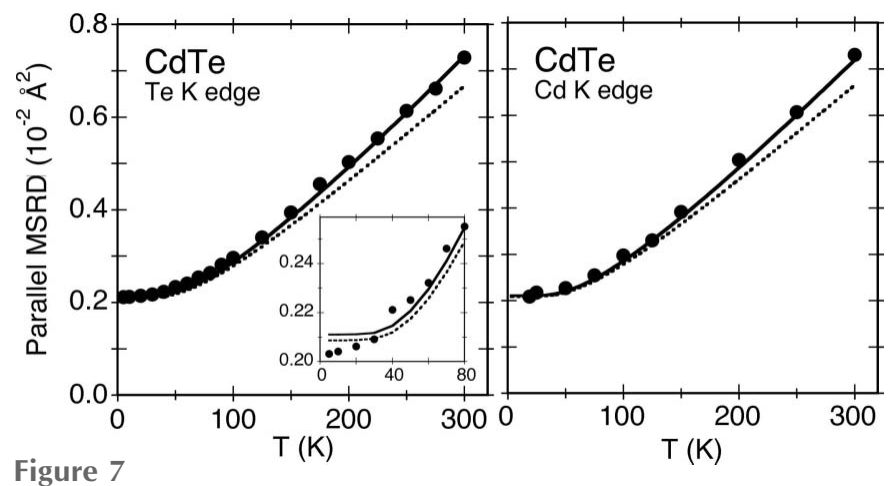

Anharmonic fit to the parallel MSRD of the first shell of CdTe (continuous line) measured at the $K$-edge of Te (left) and Cd (right); the dotted lines represent the harmonic contribution, the solid circles are experimental data.

obtained the sequence of frequencies $v_{\mathrm{E}}=3.87 \mathrm{THz}, v_{\mathrm{h}}^{(1)}=$ 4.0 $\mathrm{THz}, \ldots, v_{\text {harm }}=4.05 \mathrm{THz}$.

The final result is shown in Fig. 7 for both the Te and $\mathrm{Cd}$ edges. The harmonic contribution corresponds to $91 \%$ of the total MSRD at $300 \mathrm{~K}$ for both edges. The anharmonic contribution is mainly due to the terms (10) and (11) that depend on the $k_{3}$ force constant; the terms (9) that depend on $k_{4}$ are quite unimportant. The inset in the left panel of Fig. 7, which shows an enlarged view of the low-temperature region, allows one to evaluate the residual discrepancy between experiment and model as well as the difference between anharmonic and harmonic models, which is due to the small but not negligible quantum effects on the third cumulant.

\subsection{The bond Grüneisen parameter}

The circles and continuous lines in Fig. 7 represent the total parallel MSRD, sum of the harmonic and anharmonic contributions. According to the quasi-harmonic approximation, a harmonic Einstein model can be fit to the total MSRD value at each temperature. The frequencies $v_{\mathrm{qh}}(T)$ so obtained decrease when the temperature increases, owing to the effect of anharmonicity.

The temperature dependence $v_{\mathrm{qh}}(T)$ for the Te $K$-edge is shown in the left panel of Fig. 8 for two different values of
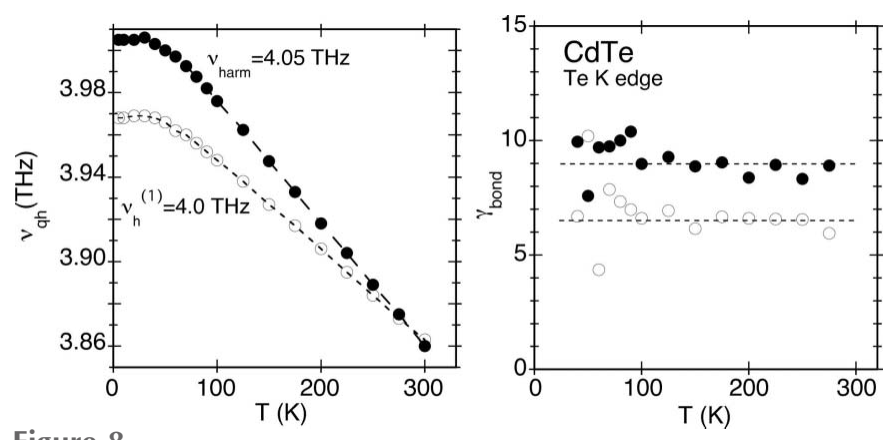

Figure 8

Temperature dependence of the quasi-harmonic Einstein frequency $v_{\mathrm{qh}}$ best fitting the total parallel MSRD (left) and of the bond Grüneisen parameters $\gamma_{\text {bond }}$ (right) for the harmonic frequencies $\nu_{\mathrm{h}}^{(1)}=4.00 \mathrm{THz}$ (open circles) and $v_{\text {harm }}=4.05 \mathrm{THz}$ (solid circles). 
harmonic contribution, $v_{\mathrm{h}}^{(1)}=4.00 \mathrm{THz}$ and $v_{\text {harm }}=4.05 \mathrm{THz}$, obtained at the first trial and at the end, respectively, of the recursive procedure depicted above. The sensitivity of the quasi-harmonic fit to tiny differences of the harmonic frequency is evident, and suggests that very high quality experimental data are necessary in order to obtain a meaningful quasi-harmonic analysis.

Within the framework of the quasi-harmonic approximation, it is reasonable to correlate the temperature dependence $v_{\mathrm{qh}}(T)$ of the frequency with the temperature dependence of the bond distance, measured by the first cumulant. To this purpose, we define a bond Grüneisen parameter

$$
\gamma_{\text {bond }}=-\frac{C_{1}^{*}}{v_{\mathrm{qh}}} \frac{\mathrm{d} v_{\mathrm{qh}}}{\mathrm{d} C_{1}^{*}}
$$

and calculate its values using the bond expansion from Abd el All et al. (2012). The results are shown in the right panel of Fig. 8. As expected, the bond Grüneisen parameter is positive, since the bond expansion is positive; one can notice how its value is strongly sensitive to tiny variations of the harmonic frequency. To the best of our knowledge, this is the first time a bond Grüneisen parameter is evaluated directly from experimental EXAFS data.

According to the unidimensional anharmonic Einstein model of Frenkel \& Rehr (1993), the value of the bond Grüneisen parameter of CdTe can be estimated as

$$
\gamma_{\text {bond }}=-\frac{3 k_{3} C_{1}^{*}}{k} \simeq 4.9 \text {, }
$$

where $k_{3}=-2.1 \mathrm{eV}^{-2}$ is the third-order force constant determined from the third cumulant (Abd el All et al., 2012) and $k=k_{\|}+6 k_{3} \delta C_{1}^{*}=3.62 \mathrm{eV} \AA^{-2}$ at $300 \mathrm{~K}$, with $k_{\|}$and $\delta C_{1}^{*}$ from experiment. The factor of three takes into account that we are here considering a linear Grüneisen parameter instead of the volume parameter considered by Frenkel \& Rehr (1993).

The volume Grüneisen parameter of CdTe evaluated at $300 \mathrm{~K}$ from the experimental coefficient of lattice thermal expansion, specific heat and compressibility is 0.6 (Novikova, 1961; Smith \& White, 1975); the same value has been recently obtained by ab initio lattice dynamics calculations (Wang et al., 2014). To compare this value with the bond Grüneisen parameter of Fig. 8, one needs to take into account that: (a) we are here considering a linear parameter instead of a volume parameter, so the value 0.6 has to be multiplied by $3 ;(b)$ the coefficient of bond expansion of CdTe at $300 \mathrm{~K}$ is $17 \times$ $10^{-6} \mathrm{~K}^{-1}$ (Fornasini \& Grisenti, 2014), about 4.2 times larger than the lattice expansion coefficient. From this procedure, a rough estimation of the bond Grüneisen parameter $\gamma_{\text {bond }} \simeq 7.6$ is obtained.

\section{Outer-shells results}

Some relevant parameters (correlation functions, force constants, Debye temperatures) of the parallel MSRD of

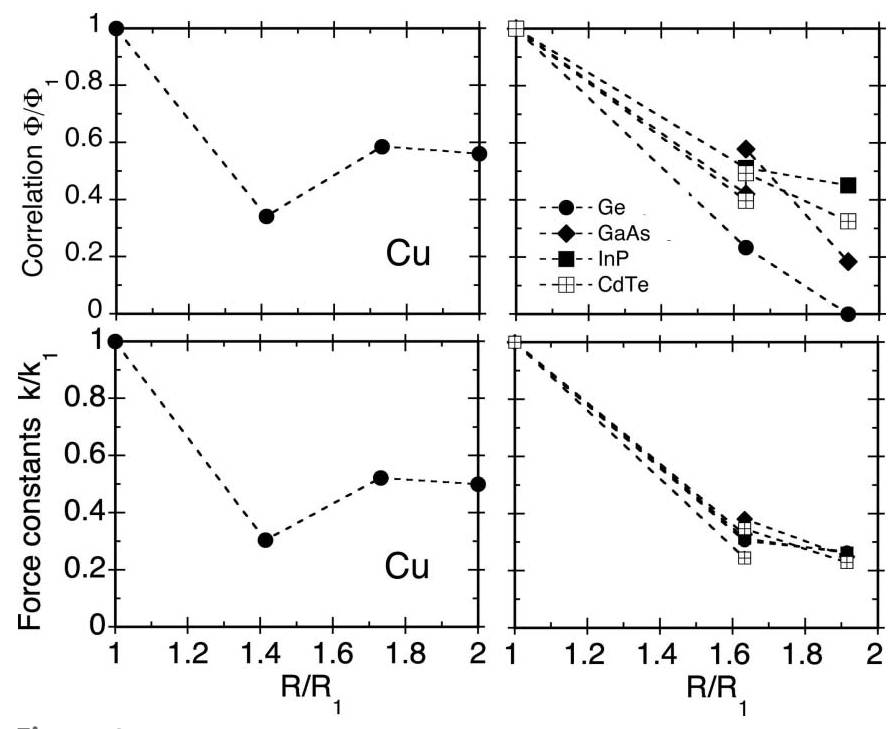

Figure 9

Correlation functions $\varphi_{\|}$at $300 \mathrm{~K}$ (top panels) and second-order force constants (bottom panels) for the outer shells of copper (left panels) and tetrahedral semiconductors (right panels), relative to the first-shell values.

outer shells in copper and several tetrahedral semiconductors are shown in Figs. 9 and 10. In all figures, the horizontal and vertical scales are normalized to the first-shell values.

\subsection{Correlation and force constants}

The value of the correlation function (24) at a given temperature (here $300 \mathrm{~K}$ ) is expected to progressively decrease in going from the first to the outer shells. In copper, where for the first shell $\varphi_{\|} \simeq 0.4$ at $300 \mathrm{~K}$, the reduction of correlation is larger for the second than for the third and fourth shells (upper left panel of Fig. 9), in agreement with the calculations for a number of other f.c.c. crystals performed by Jeong et al. (2003). In tetrahedral semiconductors, where for the first shell $\varphi_{\|} \simeq 0.8$ at $300 \mathrm{~K}$, the reduction when distance increases is more regular (upper right panel of Fig. 9), but different for different systems as well as for different secondshell pairs in the same system.
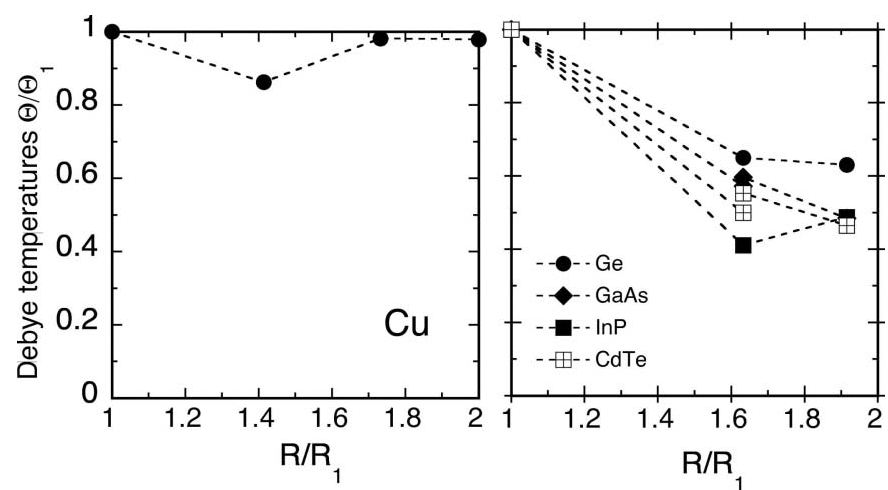

Figure 10

Debye temperatures for the outer shells of copper (left panel) and tetrahedral semiconductors (right panel), relative to the first-shell values. 
Table 1

EXAFS Debye temperatures (in K) for different shells of $\mathrm{Cu}, \mathrm{Ge}, \mathrm{GaAs}$, InP and CdTe.

For the second shells of binary compounds, the first and second lines refer to the lightest pair ( $\mathrm{Ga}-\mathrm{Ga}, \mathrm{Cd}-\mathrm{Cd}$ ) and to the heaviest pair (As-As, Te-Te, InIn), respectively. The bottom lines show the Debye temperatures from diffraction and specific heats.

\begin{tabular}{lllllll}
\hline & $\mathrm{Cu}$ & $\mathrm{Ge}$ & $\mathrm{GaAs}$ & $\mathrm{InP}$ & $\mathrm{CdTe}$ \\
\hline EXAFS & 1 & 328 & 460 & 402 & 416 & 228 \\
& 2 & 283 & 299 & 230 & & 114 \\
& 3 & 322 & 299 & 195 & 203 & 105 \\
Diffraction & 4 & 321 & & & & \\
Specific heat & $\theta_{M}$ & $320^{a}$ & $290^{b}$ & & & $143^{c, d}$ \\
& At 0 K & $347^{e}$ & $373^{e}$ & $345^{f}$ & $321^{h}$ & $158^{h}$ \\
& At 300 K & $310^{g}$ & $403^{g}$ & $370^{f}$ & $410^{h}$ & $60^{h}$ \\
& & & $350^{h}$ & & & \\
\hline
\end{tabular}

${ }^{a}$ Flinn et al. (1961). ${ }^{b}$ Batterman \& Chipman (1962). ${ }^{c}$ Zubík \& Valvoda (1975). ${ }^{d}$ Horning \& Staudenmann (1986). ${ }^{e}$ Stewart (1983). ${ }^{f}$ Adachi (1999). ${ }^{g}$ Ho et al. (1974). ${ }^{h}$ Adachi (2005).

The relative trend of the correlation functions is reproduced by the relative trend of the Einstein force constants $k_{\|}$(lower panels of Fig. 9). For the tetrahedral semiconductors, however, the relative values of the force constants are much more similar for different systems than the correlation functions. It is worth remembering that, while force constants are directly obtained from EXAFS results, correlation functions are obtained by comparing the EXAFS parallel MSRDs with the MSDs from Bragg scattering or from theoretical calculations, whose accuracy can significantly differ for different systems.

\subsection{Debye temperatures}

The Debye temperatures are listed in Table 1 and their values relative to the first shell are shown in Fig. 10.

For $\mathrm{Cu}$, the Debye temperatures are very similar for the first, third and fourth shells, and in agreement with the thermal and diffraction Debye temperatures. The Debye temperature of the second shell is, however, significantly smaller: here the Debye model cannot fully account for the reduction of correlation observed in Fig. 9, probably because of the relatively low coordination number (6) and of the consequent lack of isotropy.

For tetrahedral semiconductors, the Debye temperatures significantly decrease in going from the first to the outer shells and no connection can be found with the Debye temperatures from other techniques. Two of the assumptions of the Debye model (isotropy and correlation only attributed to inter-cell phase shifts) are clearly unsuitable for the low coordination of the first shell and for the intra-cell phase shifts of different eigenvectors.

\subsection{Anharmonicity effects}

The anharmonic analysis of the DW factor was attempted also for the second and third shells of Ge by Dalba \& Fornasini (1997). Such a procedure appears today highly unreliable, in view of the difficulty of obtaining sufficiently accurate values of third and fourth cumulants of outer shells not only by the ratio method but also by the non-linear fit approach. Moreover, as has been observed in $\$ 2$, recent simulations suggest that the outer shells are significantly less asymmetric than the first one, so that the higher-order cumulants and the anharmonic corrections to the secondorder cumulant are expected to be negligible.

\section{Conclusions}

Not only are accurate experimental evaluations of the parallel and perpendicular MSRDs in simple model systems necessary for the validation of theoretical results, their phenomenological interpretation can lead to a better intuitive understanding and to new insights on trends and regularities as a function of structural and chemical parameters.

The first-shell correlation parameters, force constants and vibrational anisotropy have different values for different structures (f.c.c. and diamond-zincblende); for the same structure, they exhibit a clear trend as a function of the bond ionicity.

Anharmonicity effects on the first-shell parallel MSRD can be evaluated, provided experimental data of very high quality are available. A quasi-harmonic analysis can then lead to evaluating a bond Grüneisen parameter. Further work, both theoretical and experimental, is necessary to assess the reliability of this procedure as well as to explore the possibility of a connection between the bond Grüneisen parameter, the coefficient of bond thermal expansion, the specific heat and the bond compressibility, in analogy with the well established relation of bulk thermodynamics.

The Debye and Einstein models can be considered equivalent, within the present-day experimental accuracy, for a simple parametrization of the temperature dependence of parallel and perpendicular MSRDs. The example of CdTe suggests that the Einstein model can give a slightly better estimate of the absolute values for non-primitive crystals. The EXAFS Debye temperatures cannot generally be compared with the Debye temperatures from other techniques; their values can in any case give the qualitative separation of classical from quantum regimes for the different coordination shells. The Einstein model is recommended for its simplicity, for the possibility of evaluation of the effective force constants and for the easyness of extension to include anharmonicity effects.

\section{Acknowledgements}

This paper is the result of a long-term experimental activity conducted at different synchrotron radiation beamlines (ESRF-BM08, ESRF-BM23, Elettra-XAFS) in cooperation with a number of people, to which the authors are deeply indebted: G. Dalba, F. Rocca, N. Abd el All, S. Ahmed, M. Vaccari, A. Sanson, J. Purans, A. Kuzmin, D. Diop and B. Thiodjio Sendja. The authors are grateful to T. Miyanaga, T. Fujikawa, J. Rehr and F. Vila for helpful and stimulating 
discussions. C. Schnohr is acknowledged for communicating numerical data of InP.

\section{References}

Abd el All, N., Dalba, G., Diop, D., Fornasini, P., Grisenti, R., Mathon, O., Rocca, F., Thiodjio Sendja, B. \& Vaccari, M. (2012). J. Phys. Condens. Matter, 24, 115403.

Abd el All, N., Thiodjio Sendja, B., Grisenti, R., Rocca, F., Diop, D., Mathon, O., Pascarelli, S. \& Fornasini, P. (2013). J. Synchrotron Rad. 20, 603-613.

a Beccara, S., Dalba, G., Fornasini, P., Grisenti, R., Pederiva, F., Sanson, A., Diop, D. \& Rocca, F. (2003). Phys. Rev. B, 68, 140301. a Beccara, S. \& Fornasini, P. (2008). Phys. Rev. B, 77, 172304.

Adachi, S. (1999). GaAs and Related Materials. Singapore: World Scientific.

Adachi, S. (2005). Properties of Group IV, III-V and II-VI Semiconductors. New York: Wiley.

Agostini, G., Piovano, A., Bertinetti, L., Pellegrini, R., Leofanti, G., Groppo, E. \& Lamberti, C. (2014). J. Phys. Chem. C, 118, 40854094.

Ahmed, S. I., Aquilanti, G., Novello, N., Olivi, L., Grisenti, R. \& Fornasini, P. (2013). J. Chem. Phys. 139, 164512.

Ahmed, S. I., Dalba, G., Fornasini, P., Vaccari, M., Rocca, F., Sanson, A., Li, J. \& Sleight, A. W. (2009). Phys. Rev. B, 79, 104302.

Altorfer, F., Graneli, B., Fischer, P. \& Buhrer, W. (1994). J. Phys. Condens. Matter, 6, 9949-9962.

Ankudinov, A. L., Ravel, B., Rehr, J. J. \& Conradson, S. D. (1998). Phys. Rev. B, 58, 7565-7576.

Araujo, L. L., Giulian, R., Sprouster, D. J., Schnohr, C. S., Llewellyn, D. L., Kluth, P., Cookson, D. J., Foran, G. J. \& Ridgway, M. C. (2008). Phys. Rev. B, 78, 094112.

Artioli, G. (2002). EMU Notes in Mineralogy, Vol. 4, Energy Modelling in Minerals, edited by C. M. Gramaccioli, pp. 389-405. Budapest: Eötvös University Press.

Ascone, I., Asakura, K., George, G. N. \& Wakatsuki, S. (2012). J. Synchrotron Rad. 19, 849-850.

Batterman, B. W. \& Chipman, D. R. (1962). Phys. Rev. 127, 690-693.

Benfatto, M., Natoli, C. R. \& Filipponi, A. (1989). Phys. Rev. B, 40, 9626-9635.

Beni, G. \& Platzman, P. M. (1976). Phys. Rev. B, 14, 1514-1518.

Bohmer, W. \& Rabe, P. (1979). J. Phys. C, 12, 2465-2474.

Booth, C. H., Bridges, F., Bauer, E. D., Li, G. G., Boyce, J. B., Claeson, T., Chu, C. W. \& Xiong, Q. (1995). Phys. Rev. B, 52, R15745R15748.

Bridges, F., Keiber, T., Juhas, P., Billinge, S. J. L., Sutton, L., Wilde, J. \& Kowach, G. (2014). Phys. Rev. Lett. 112, 045505.

Bunker, G. (1983). Nucl. Instrum. Methods Phys. Res. 207, 437444.

Busing, W. R. \& Levy, H. A. (1964). Acta Cryst. 17, 142-146.

Cochran, W. (1971). Acta Cryst. A27, 556-559.

Comaschi, T., Balerna, A. \& Mobilio, S. (2008). Phys. Rev. B, 77, 075432.

Crozier, E. D., Rehr, J. J. \& Ingalls, R. (1988). In X-ray Absorption, edited by D. C. Koningsberger and R. Prins, ch. 9, pp. 373-442. New York: J. Wiley and Sons.

Dalba, G. \& Fornasini, P. (1997). J. Synchrotron Rad. 4, 243-255.

Dalba, G., Fornasini, P., Gotter, R. \& Rocca, F. (1995a). Phys. Rev. B, 52, 149-157.

Dalba, G., Fornasini, P., Grazioli, M. \& Rocca, F. (1995b). Phys. Rev. $B, \mathbf{5 2}, 11034-11043$.

Dalba, G., Fornasini, P., Grisenti, R., Pasqualini, D., Diop, D. \& Monti, F. (1998). Phys. Rev. B, 58, 4793-4802.

Dalba, G., Fornasini, P., Grisenti, R. \& Purans, J. (1999). Phys. Rev. Lett. 82, 4240-4243.

Dalba, G., Fornasini, P. \& Rocca, F. (1993). Phys. Rev. B, 47, 85028514.
Dalba, G., Fornasini, P., Rocca, F. \& Mobilio, S. (1990). Phys. Rev. B, 41, 9668-9675.

Day, J. T., Mullen, J. G. \& Shukla, R. (1995). Phys. Rev. B, 52, 168176.

Dimakis, N. \& Bunker, G. (1998). Phys. Rev. B, 58, 2467-2475.

Edwards, A. B., Tildesley, D. J. \& Binsted, N. (1997). Mol. Phys. 91, 357-369.

Eisenberger, P. \& Brown, G. S. (1979). Solid State Commun. 29, 481484.

Feynman, R. P. (1972). Statistical Mechanics. Reading, MA: Benjamin.

Filipponi, A. (2001). J. Phys. Condens. Matter, 13, R1-R38.

Flinn, P. A., McManus, G. M. \& Rayne, J. A. (1961). Phys. Rev. 123, 809-812.

Fornasini, P. (2001). J. Phys. Condens. Matter, 13, 7859-7872.

Fornasini, P. (2015a). Synchrotron Radiation, edited by S. Mobilio, F. Boscherini \& C. Meneghini, pp. 181-211. Berlin/Heidelberg: Springer.

Fornasini, P. (2015b). In X-ray Absorption Spectroscopy of Semiconductors, edited by C. S. Schnohr \& M. C. Ridgway, ch. 6, pp. 127-141. New York: Springer.

Fornasini, P., a Beccara, S., Dalba, G., Grisenti, R., Sanson, A., Vaccari, M. \& Rocca, F. (2004). Phys. Rev. B, 70, 174301.

Fornasini, P., el All, N. A., Ahmed, S. I., Sanson, A. \& Vaccari, M. (2009). J. Phys. Conf. Ser. 190, 012025.

Fornasini, P. \& Grisenti, R. (2014). J. Chem. Phys. 141, 164503.

Fornasini, P., Monti, F. \& Sanson, A. (2001). J. Synchrotron Rad. 8, 1214-1220.

Frenkel, A. I. \& Rehr, J. J. (1993). Phys. Rev. B, 48, 585-588.

Freund, J., Ingalls, R. \& Crozier, E. D. (1989). Phys. Rev. B, 39, $12537-$ 12547.

Fujikawa, T. \& Miyanaga, T. (1993). J. Phys. Soc. Jpn, 62, 4108-4122. Haug, J., Chassé, A., Schneider, R., Kruth, H. \& Dubiel, M. (2008). Phys. Rev. B, 77, 184115.

Ho, C. Y., Powell, R. W. \& Liley, P. E. (1974). J. Phys. Chem. Ref. Data, 3(Suppl. 1), 1-796.

Horning, R. D. \& Staudenmann, J. L. (1986). Phys. Rev. B, 34, 3970 3979.

Ikemoto, H. \& Miyanaga, T. (2007). Phys. Rev. Lett. 99, 165503.

Jeong, I. K., Heffner, R. H., Graf, M. J. \& Billinge, S. J. L. (2003). Phys. Rev. B, 67, 104301.

Jeong, I. K., Proffen, T., Mohiuddin-Jacobs, F. \& Billinge, S. J. L. (1999). J. Phys. Chem. A, 103, 921-924.

Kamishima, O., Ishii, T., Maeda, H. \& Hashino, S. (1997a). Solid State Commun. 103, 141-144.

Katsumata, H., Miyanaga, T., Yokoyama, T., Fujikawa, T. \& Ohta, T. (2001). J. Synchrotron Rad. 8, 226-228.

Keating, P. N. (1966). Phys. Rev. 145, 637-645.

Kennedy, B. J. (1995). Acta Cryst. C51, 790-792.

Lagarde, P. (1985). Amorphous Solids and the Liquid State, edited by N. H. March, R. A. Street and M. Tosi, ch. 11, pp. 365-394. New York: Plenum Press.

Lee, P. A., Citrin, P. H., Eisenberger, P. \& Kincaid, B. M. (1981). Rev. Mod. Phys. 53, 769-806.

Martin, C. J. \& O'Connor, D. A. (1977). J. Phys. C, 10, 3521-3526.

Martin, R. M. (1971). Phys. Rev. B, 1, 4005-4011.

Miyanaga, T. \& Fujikawa, T. (1994). J. Phys. Soc. Jpn, 63, 1036-1052. Miyanaga, T. \& Fujikawa, T. (1998). J. Phys. Soc. Jpn, 67, 2930-2937.

Mustre de Leon, J., Conradson, S. D., Batistić, I., Bishop, A. R., Raistrick, I. D., Aronson, M. C. \& Garzon, F. H. (1992). Phys. Rev. $B, 45,2447-2457$.

Newville, M. (2001). J. Synchrotron Rad. 8, 322-324.

Novikova, S. I. (1961). Sov. Phys. Solid State, 2, 2087-2089.

Poiarkova, A. V. \& Rehr, J. J. (1999). Phys. Rev. B, 59, 948-957.

Popescu, C., Itie, J., Congedutti, A., Lagarde, P., Flank, A., PinsardGaudart, L. \& Dragoe, N. (2011). Phys. Rev. B, 84, 224120.

Principi, E., Di Cicco, A., Decremps, F., Polian, A., De Panfilis, S. \& Filipponi, A. (2004). Phys. Rev. B, 69, 201201. 
Purans, J., Afify, N. D., Dalba, G., Grisenti, R., De Panfilis, S., Kuzmin, A., Ozhogin, V. I., Rocca, F., Sanson, A., Tiutiunnikov, S. I. \& Fornasini, P. (2008). Phys. Rev. Lett. 100, 055901.

Ravel, B. \& Newville, M. (2005). J. Synchrotron Rad. 12, 537-541.

Rehr, J. J. \& Alben, R. (1977). Phys. Rev. B, 16, 2400-2407.

Rehr, J. J. \& Albers, R. C. (2000). Rev. Mod. Phys. 72, 621-654.

Rehr, J. J., Albers, R. C. \& Zabinsky, S. I. (1992). Phys. Rev. Lett. 69, 3397-3400.

Reid, J. S. (1983). Acta Cryst. A39, 1-13.

Ruffoni, M. P., Pettifer, R. F., Pascarelli, S., Trapananti, A. \& Mathon, O. (2007). J. Synchrotron Rad. 14, 421-425.

Sanson, A. (2008). J. Synchrotron Rad. 15, 514-518.

Sanson, A. (2010). Phys. Rev. B, 81, 012304.

Sanson, A., Armellini, C., Grisenti, R. \& Fornasini, P. (2013). J. Phys. Chem. C, 117, 6081-6087.

Sanson, A., Rocca, F., Dalba, G., Fornasini, P., Grisenti, R., Dapiaggi, M. \& Artioli, G. (2006). Phys. Rev. B, 73, 214305.

Saravanan, R., Mohanlal, S. K. \& Chandrasekaran, K. S. (1992). Z. Kristallogr. 200, 7-13.

Schnohr, C. S., Araujo, L. J. \& Ridgway, M. C. (2014). J. Phys. Soc. Jpn, 83, 094602.

Schnohr, C. S., Kluth, P., Araujo, L. L., Sprouster, D. J., Byrne, A. P., Foran, G. J. \& Ridgway, M. C. (2009). Phys. Rev. B, 79, 195203.

Sevillano, E., Meuth, H. \& Rehr, J. J. (1979). Phys. Rev. B, 20, 4908 4911.

Smith, T. F. \& White, G. K. (1975). J. Phys. C, 8, 2031-2042.

Soma, T. \& Matsuo, H. (1982). Phys. Status Solidi B, 111, K93-K97.

Stern, E. A. (1997). J. Phys. IV Fr. 7, 137-140.

Stern, E. A., Livnņš, P. \& Zhang, Z. (1991). Phys. Rev. B, 43, 88508860.

Stewart, G. R. (1983). Rev. Sci. Instrum. 54, 1-11.

Talwar, D. N. \& Holliday, K. (1999). Physica B, 263-264, 540-545.
Tranquada, J. M. \& Ingalls, R. (1983). Phys. Rev. B, 28, 3520-3528.

Vaccari, M. (2006). PhD thesis, University of Trento, Italy.

Vaccari, M. \& Fornasini, P. (2005). Phys. Rev. B, 72, 092301.

Vaccari, M. \& Fornasini, P. (2006). J. Synchrotron Rad. 13, $321-$ 325.

Vaccari, M., Grisenti, R., Fornasini, P., Rocca, F. \& Sanson, A. (2007). Phys. Rev. B, 75, 184307.

Van Hung, N. \& Fornasini, P. (2007). J. Phys. Soc. Jpn, 76, 084601.

Van Hung, N. \& Rehr, J. J. (1997). Phys. Rev. B, 56, 43-46.

Van Hung, N., Sy Thang, C., Cong Toan, N. \& Khac Hieu, H. (2014b). Vacuum, 101, 63-66.

Van Hung, N., Sy Tien, T., Ba Duc, N. \& Quoc Vuong, D. (2014a). Mod. Phys. Lett. 28, 1450174.

Vila, F. D., Lindahl, V. E. \& Rehr, J. J. (2012). Phys. Rev. B, 85, 024303.

Vila, F. D., Rehr, J. J., Rossner, H. H. \& Krappe, H. J. (2007). Phys. Rev. B, 76, 014301.

Wang, L., Yuan, P.-F., Wang, F., Sun, Q., Guo, Z.-X., Liang, E.-J. \& Jia, Y. (2014). Mater. Chem. Phys. 148, 214-222.

Willis, B. T. M. \& Pryor, A. W. (1975). Thermal Vibrations in Crystallography. Cambridge University Press.

Yang, D. S., Fazzini, D. R., Morrison, T. I., Tröger, L. \& Bunker, G. (1997). J. Non-Cryst. Solids, 210, 275-286.

Yokoyama, T. (1998). Phys. Rev. B, 57, 3423-3432.

Yokoyama, T. (1999). J. Synchrotron Rad. 6, 323-325.

Yokoyama, T. (2004). Private communication.

Yokoyama, T. \& Eguchi, K. (2011). Phys. Rev. Lett. 107, 065901.

Yokoyama, T., Kobayashi, K., Ohta, T. \& Ugawa, A. (1996). Phys. Rev. B, 53, 6111-6122.

Yokoyama, T., Ohta, T. \& Sato, H. (1997). Phys. Rev. B, 55, 11320 11329.

Zubík, K. \& Valvoda, V. (1975). Czech. J. Phys. 25, 1149-1154. 